\title{
An investigation of surface stress on the fracture mechanics behavior of classical and phase-separating planar electrodes
}

\author{
Seyed Ali Mahmoudy, Hamed Haftbaradaran \\ Department of Civil Engineering, University of Isfahan, Isfahan, Iran
}

\begin{abstract}
Although lithium-ion batteries have extensively been used in various applications because of their high energy capacity, fracture and failure, the by-products of large strains and stresses caused by fast charging and discharging need yet to be addressed. The size effects on the mechanical behavior of the nano-sized structures are significant; however, the classical elasticity theory may not consider such effects. On the other hand, surface stress theory, as a robust and potential theory, is suitable in considering size effects in nano-scale structures. Therefore, in this paper, in order to involve the surface stress effects on the fracture behavior of Li-ion batteries, the following steps are taken. Firstly, a phase-field model is used to determine the evolution of the concentration profile. Subsequently, the stress distribution is obtained by using the surface stress theory combined with chemical equations for a planar electrode. Afterward, by using the weight function method for an edge crack in the plate, the stress intensity factor is derived for all time steps and possible crack lengths during the process. It is found that with increasing phase boundary thickness parameter or decreasing phase-separation phenomenon, the surface mechanics parameters become more influential. Furthermore, in the presence of positive surface stress, the diffusion-induced stress distribution decreases, which in turn reduces the stress intensity factor. In addition, in this paper, the two states of surface stress are compared either for elastic or total strain. Concerning stresses and concentrations, the results indicate a big difference at the beginning of the deintercalation process showing, in particular, $2 \%$ for stresses, but the differences diminish gradually.
\end{abstract}

Keywords: Lithium-ion batteries; phase-separation; fracture mechanics; surface stress; diffusion-induced stresses

*Corresponding author

E-mail address: A.Mahmoudy@trn.ui.ac.ir; alimah35@gmail.com 


\section{Introduction}

Lithium-ion batteries have been widely used in various applications because of their great potentials and advantages [1]. Capability of storing high-density energy is a significant advantage, however, fast diffusion in such batteries can result in high stresses and strains, which can lead to cracking [2], debonding [3], yielding [4] and other types of failure. Therefore, understanding the mechanical behavior of lithium-ion batteries has been paid particular attention by researchers. The performance of lithium-ion batteries can be improved through various methods, e.g. employing nanostructures [5], core-shell structures [6], and surface coatings [7].

Experiments showed that the response of the battery to mechanical loads is size-dependent in micro and nanoscale. However, such responses cannot be characterized by classical elasticity theories [8], [9]. Therefore, size-dependent continuum theories have been developed to take into account these small-scale effects. Nonlocal elasticity [10], couple stress theory [11], micropolar elasticity [12], strain gradient theory [13] and surface stress theory $[14,15]$ are the examples of continuum theories that have been investigated in the literature.

Several studies have been dedicated to understanding the diffusion-induced stresses caused by lithiation and delithiation in the electrode particles. By using thermal stress analogy, Prussin [16] determined the transverses stresses imposed on a thin plate during mass transfer. Christensen and Newman [17] proposed an analytical solution to determine the maximum particle stress as a function of dimensionless current, which includes the charge rate, particle size, and diffusion coefficient. In addition, the effects of pressure-driven diffusion and nonideal interactions between the lithium and host material were studied in several studies. Cheng and Verbrugge [18] acquired the analytic concentration, stress distribution, and strain energy density for spherical particles under galvanostatic and potentiostatic conditions. The results showed that the corresponding 
stresses associated with the two conditions were quite different. Zhang et al. [19] studied the stresses in spherical and elliptical particles using the Finite Element Method by considering the effect of hydrostatic stress on diffusion. The results showed that smaller particles with larger aspect ratios were favorable in terms of diffusion-induced stresses. Peng et al. [20] provided semianalytical solutions for different lithium concentrations and stresses in the finite cylindrical electrode under traction free conditions and potentiostatic operation. The results showed a good agreement between numerical and simulation results for the distribution of lithium concentration while a slight difference for the radial and hoop stresses was observed.

Experimental and analytical studies have shown that during the lithiation of lithium-ions, a phase separation might occur, which was reported in Refs. [21-23] for $\mathrm{Li}_{\mathrm{y}} \mathrm{Mn}_{2} \mathrm{O}_{4}$. Accordingly, some phases could emerge; as a result, further structural distortions, volumetric changes, mechanical stresses and strains could appear [24]. Park et al. [25] proposed a three-dimensional finite element model to calculate the concentration and stresses resulted from phase transition for lithium manganese dioxide particles with arbitrary geometries. The outcomes showed that the stresses were contingent on particle geometry, lithium diffusivity and input current density. In addition, phase transitions were observed to contribute to the stresses more than other parameters. Deshpande et al. [26] calculated diffusion-induced stresses in phase transforming electrodes using a core-shell structural model in which the abrupt transitions in concentrations at phase boundaries contributed to stress discontinuities. In their research, the effects of mechanical properties of two phases were investigated on stress formation, stress discontinuity and strain energy. Song et al. [27] proposed a phase-field model to study the effects of phase separation on concentration and diffusion-induced stresses in a planar electrode. The influences of energies and charge operation were investigated showing that the phase separation profile was more influential on concentration 
and stresses compared to charge operation. Xie et al. [28] investigated the influence of the diffusion process, structural deformation and local stress-strain evolution on the geometry of a 2D silicon nanowire using a phase-field model. The numerical results showed that large local stresses were created in the outer region and also lithiation depth of the electrode was limited by small lithium mobility. In addition, a high charging rate caused high stresses in the vicinity of the silicon nanowire core region.

Size effects of nanoparticles also play a critical role in the performance of lithium-ion batteries. Several studies have attempted to apply surface stress theory to energy storage materials and lithium-ion batteries. A general chemo-mechanics theory for elastic solids with surface stress was proposed by Gao et al. [29]. However, for lithium-ion batteries, in particular, Cheng and Verbrugge [30] have shown that both the magnitude and distribution of stresses can be significantly affected by surface mechanics for nanoscale particles. Deshpande et al. [31] concluded that diffusion-induced stresses, especially the tensile stresses, can significantly decrease due to the surface effects. Hao et al. [32] investigated the surface effects on solid and hollow electrode nanoparticles. They also showed that hollow electrode particles were superior to solid electrode particles in terms of mechanical behavior. Hao and Fang [33] developed a model for diffusion-induced stresses in which the effects of surface stress were involved. It was concluded that the carbon shell and residual surface tension reduced tensile stresses within the core electrode particles, which could prevent brittle fracture. Liu et al. [34] developed a model to investigate the effects of hydrostatic stress, surface stress, phase transformation, and structure of electrodes; meanwhile, the smaller and hollow particles were shown to be beneficial in terms of stresses. They concluded that when the effects of hydrostatic stress were taken into account, the phase transformation process occurred more rapidly. Zhang et al. [35] investigated the effects of surface 
stress, stress dependent chemical potential and stress mediated diffusivity on lithium diffusion kinetics. The results showed that with decreasing the particle size, less lithiation was attained inside the electrode in which the lithium ions were accumulated in the vicinity of surface region because of larger compressive stress caused by surface stress. Shodja et al. [36] obtained analytical diffusion-induced stresses for a core-shell nano-structure under galvanostatic operation using surface elasticity theory. They showed that these values of stress depend on the different modes of charging.

In addition, the fracture mechanics behavior of lithium-ion batteries has hardly been studied and understood. Taking into account the fracture mechanics, Hu et al. [37] studied the critical situation of crack propagation in a phase-separating $\mathrm{LiFePO}_{4}$ particle. They used fracture mechanics to estimate the maximal rate of energy discharge among various cracks created by the incongruity between phases in $\mathrm{LiFePo}_{4}$. Zhao et al. [38] calculated the inhomogeneous distribution of lithium and studied stress-related factors in addition to estimating the energy release rate for a $\mathrm{LiCoO}_{2}$ particle containing preexisting cracks. They predicted the critical rate of charging and size of the particle below which the fracture was averted. Woodford et al. $[39,40]$ used fracture mechanics to predict the fracture of electrode particles. The results for anisotropic particles showed that reducing the maximum shear strain would avoid the unfavorable fracture behavior of particles. In addition, they plotted fracture toughness, rate of diffusion, and particle size to select and design electrodes in safe areas. In another work, the results of their previous study for phase separating electrode particles were confirmed [24]. Zhao et al. [41] studied the fracture and debonding failure in coated hollow spherical particles and silicon anode nanowires. They investigated critical structural parameters and operating conditions to avoid fracture of the coating and debonding between the coating and the active material. Chen et al. [42] investigated the effect of phase transformation on 
concentration distribution and stress evolution where sharp drop hoop stress and lithium-ion concentration were observed at the interface between phases. Then, they calculated the stress intensity factor for an interfacial crack in a two-phase separating spherical electrode particle subjected to galvanostatic charging. Additionally, they plotted failure mechanics criterion based on critical nanoparticle electrode size and current density, and realized that the SIF will decrease with reducing the particle size and charging time. Haftbaradaran et al. [43] employed a phasefield model to predict the way of phase separation contribution to the evolution of the concentration and stress profile in a planar electrode. Following the fracture mechanics approach, they obtained the stress intensity factors for a centeral crack and cracks forming at the edge, separately in a planer electrode during insertion and extraction half-cycles and derived a critical electrode thickness. Esmizadeh et al. [44] studied the fracture mechanics of two-phase separating cylindrical and spherical electrode particles with core-shell structures subjected to deintercalation half-cycle. They obtained stress intensity factor for the preexisting surface crack and critical particle size to avoid fracture of particles in the presence of high and low surface flux. Movahedi et al. [45] studied fracture mechanics of two-phase electrode particles with different elastic moduli of each phases. The stress intensity factors and energy release rates were calculated for center and edge cracks and the effects of small and large surface fluxes on the energy release rates were investigated. Hu et al. [46] obtained the diffusion-induced stress and stress intensity factor for an edge crack in the presence of surface stress for nano-spherical electrode particles. The results showed that the diffusion-induced stress decreased due to surface effects and the stress intensity factor increased monotonically with charging time and crack length. Finally, they derived the critical electrode particle size in which the particles of smaller size were not ruptured and was shown that nanoparticles were more resilient to fracture. $\mathrm{Xu}$ et al. [47] determined the critical sizes of a 
cylindrical anode and showed that a radial crack is more critical than a circumferential crack for a hollow cylindrical anode. It was attributed to the lower stress intensity factor for circumferential crack compared to that of the radial crack in the presence of surface stress.

To the best of our knowledge, no research has been conducted to evaluate the combined effect of surface stresses and phase separation phenomenon on the fracture mechanics behavior of electrode particles. Therefore, the main novelties and objectives of this paper can be summarized as:

- To evaluate the effects of surface mechanics parameters on the concentration, diffusioninduced stresses, and stress intensity factor for an edge cracked plate under deintercalation process.

- Two states of surface stress for elastic strain or total strain are compared with regard to diffusion-induced stresses.

- Phase separation parameters and surface stress parameters are compared concerning diffusion-induced stresses and stress intensity factor.

\section{Methodology}

This study investigates the effects of surface mechanics and phase-separating parameters on the stress distribution and fracture behavior of a planar electrode. In order to address the problem, the following steps are taken:

1. Obtaining the concentration distribution considering the effect of phase-separation by combining the mass diffusion and phase-field model.

2. The stress distribution is obtained analytically based on elasticity equations, which depends on concentration values and surface stress parameters. 
3. The stress intensity factor is calculated using the weight function method and the effects of geometry, loading and other effective parameters on crack growth is discussed.

\subsection{Problem statement and governing equations}

Considering a free-standing rectangular planar electrode with the thickness of $2 h$ subjected to uniform solute extraction in addition to free surfaces at $x= \pm h$ as shown in Fig. 1. The dimensions of the electrode in $y$ and $z$ directions are assumed to be considerably larger than its thickness, and solute exchange with the surroundings happens uniformly at each point along the electrode surface. Therefore, a one-dimensional problem can be considered as a reasonable assumption.

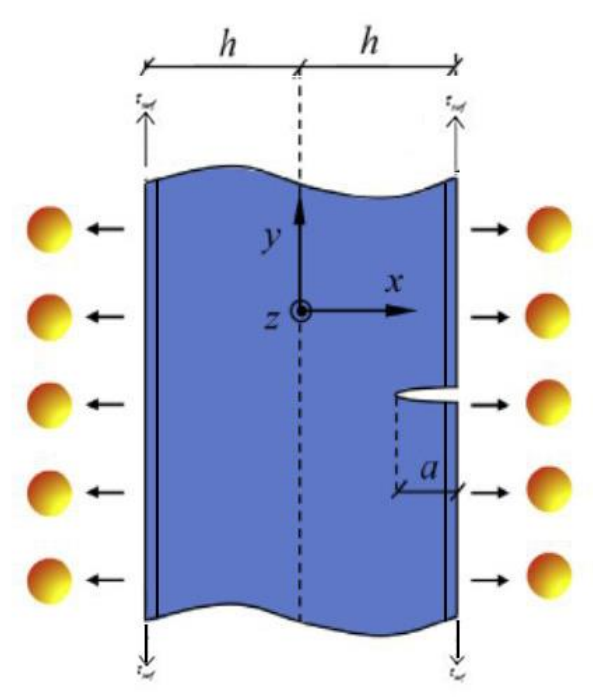

Fig. 1: Schematic of a planar electrode with the thickness of $2 \mathrm{~h}$ subjected to deintercalation in the presence of surface stress

\subsubsection{Mass diffusion}

The equation of mass conservation for solute diffusion in the host material can be written as follows [48]: 
$\frac{\partial c}{\partial t}+\frac{\partial j}{\partial x}=0$

where $c$ denotes the solute concentration (in terms of mole per unit volume of the solution), and $j$ is the atomic flux, which relates to the thermodynamic diffusional potential $\mu$ given by [48]:

$j=-M c \frac{\partial \mu}{\partial x}$

here, $M$ is the mobility coefficient. According to Refs. [47,48], it is assumed that the mobility increases linearly with the concentration of the unoccupied interstitial sites:

$M=M_{0}\left[1-\frac{c}{c_{\max }}\right]$

where $M_{0}$ refers to the mobility of the solute in the solute-free host (i.e., with $c=0$ ) when all interstitial sites are unoccupied and $c_{\max }$ is the saturation limit of the solution corresponding to a case where all interstitial sites are occupied with the guest atoms.

\subsubsection{Phase-field model}

In a phase-field model, the total free energy of a solution consists of three parts [49]

$F=\int_{V}\left(f_{h}+f_{g}+f_{e l}\right) d V$

$f_{h}=\omega c\left(1-\frac{c}{c_{\max }}\right)+R T\left(c \log \frac{c}{c_{\max }}+\left(c_{\max }-c\right) \log \left(1-\frac{c}{c_{\max }}\right)\right)$

$f_{g}=\frac{1}{2} k_{g}(\nabla c)^{2}$

where $f_{h}$ is the homogenous free energy, $f_{g}$ is the gradient energy, $f_{e l}$ is the elastic strain energy, and $V$ is the volume of the solution. $\omega$ shows the strength of interaction between the solute and 
the host, and $R$ and $T$ are the universal gas constant and absolute temperature, respectively. $k_{g}$ is the gradient energy coefficient accounting for the free energy of the phase boundary and $\nabla c$ is the gradient of concentration. For the sake of simplicity and the reason mentioned in Ref. [44], the elastic strain energy is not considered.

Using Eq. (4), the diffusional chemical potential $\mu$ is obtained by taking the variational derivative of $F$ to $c$ [50] as given below:

$\mu=\frac{\delta F}{\delta c}=\frac{\partial f_{h}}{\partial c}-k_{g} \nabla^{2} c$

After combining Eqs. (1)-(7), a first-order nonlinear partial differential equation with respect to time and fourth-order with regard to the space is obtained for the evolution of the concentration distribution in the electrode. For the simplicity, the dimensionless form of the equations is derived considering the initial and natural boundary conditions.

$\frac{\partial \tilde{c}}{\partial \tau}=\frac{\partial}{\partial \tilde{x}}\left[(1-2 \tilde{\omega} \tilde{c}(1-\tilde{c})) \frac{\partial \tilde{c}}{\partial \tilde{x}}-\left(\frac{\lambda}{h}\right)^{2} \tilde{c}(1-\tilde{c}) \frac{\partial^{3} \tilde{c}}{\partial \tilde{x}^{3}}\right]$

$\tilde{c}=\tilde{c_{i}}$ at $\tau=0$

$\tilde{j}=\tilde{j}_{0}(1-\tilde{c})^{0.5} \tilde{c}^{0.5}, \frac{\partial \tilde{c}}{\partial \tilde{x}}=0$, at $\tilde{x}=1$

$\tilde{j}=0, \frac{\partial \tilde{c}}{\partial \tilde{x}}=0$, at $\tilde{x}=0$

$\tilde{j}, \tau, \tilde{x}, \tilde{c_{i}}, \tilde{\omega}$ and $\tilde{j}_{0}$ are defined as Eqs. (12) to (18), respectively.

$\tilde{j}=-(1-2 \tilde{\omega} \tilde{c}(1-\tilde{c})) \frac{\partial \tilde{c}}{\partial \tilde{x}}+\left(\frac{\lambda}{h}\right)^{2} \tilde{c}(1-\tilde{c})\left(\frac{\partial^{3} \tilde{c}}{\partial \tilde{x}^{3}}\right)$ 
$\tau=\left(\frac{D_{0} t}{h^{2}}\right)$

$\tilde{x}=\frac{x}{h}$

$\tilde{c_{i}}=\frac{c_{i}}{c_{\max }}$

$\tilde{\omega}=\frac{\omega}{R T}$

$\tilde{j}_{0}=\frac{j_{0} h}{D_{0} c_{\max }}$

$D_{0}=M R T$ is the diffusivity coefficient in the limit of dilute solution and $\lambda=\sqrt{\left(\frac{k_{g} c_{\max }}{R T}\right)}$ is the natural length scale of the problem, which sets the width of the interphase region and is typically in the order of nanometers $[51,52]$.

The first part of Eq. (10) comes from Butler-Volmer equation and is derived excluding volume effects [55] and the surface overpotential parameter at the electrode surface is assumed to be constant regardless of the solute concentration at the electrode surface. The second part of Eq. (10) is a natural boundary condition in which the surface energy is constant which does not depend on the surface solute concentration $[54,55]$. Eq. (11) is written using the symmetry of the problem with respect to $\tilde{x}=0$.

Using the central finite difference method, we discretize the nonlinear equations over the domain $0 \leq \tilde{x} \leq 1$

It should be noted that by setting $\tilde{\omega}$ equal to zero and neglecting the higher order derivative term in Eq. (8), the usual Fickian diffusion equation is derived. 


\subsection{Diffusion induced stresses (DIS)}

Because of the boundary constraints and inhomogeneous concentration distribution, the planar electrode is subjected to stresses that vary along the width of the particle in $x$ direction and with time. The obtained diffusion-induced stress equation is based on elasticity equations including equilibrium, stress-strain relation, and compatibility equations. After deriving the DIS equation, firstly, it is required to determine the concentration distribution, which is obtained numerically for limited nodes in the previous section based on finite difference method. After that, the diffusioninduced stress values considering time variant surface stress are calculated for the same nodes, e.g., concentration distribution.

Initially, the concept of surface stress is explained, subsequently, the diffusion-induced stress distribution in the presence of surface stress would be calculated and compared with the regular situation excluding surface stress.

\subsubsection{Surface stresses}

Surface effects play a crucial role in nano-structures due to their high surface to volume ratio. In other words, for nanomaterials, the energy of atoms that are placed on the outer surface of the rigid body is different from those placed in the interior bulk. Gibbs [58] introduced the concept of surface energy and surface stress in which the expression for surface stress tensor is written as follows:

$$
\sigma_{\alpha \beta}^{s u f f}=\gamma \delta_{\alpha \beta}+\frac{\partial \gamma}{\partial \varepsilon_{\alpha \beta}^{s}}
$$

where $\gamma$ is surface energy per unit area, $\varepsilon_{\alpha \beta}^{s}$ is surface strain tensor $(\alpha, \beta=1,2)$ and $\delta_{\alpha \beta}$ denotes Kronecker delta function. Besides, the physical meaning of these quantities with theoretical calculation and experimental measurements were given in Ref. [56], and the concepts were 
extended and developed in Refs. [57-60]. The surface stress and $\gamma$ have different values for solid boundaries contrary to liquids; because of the high mobility of atoms in fluids.

A general theory of surface elasticity has been developed by Gurtin and Murdoch $[14,15]$ in which an isotropic membrane layer as a surface with zero thickness is assumed to adhere to the bulk without any friction. In this condition, two sets of equations for layer and bulk should be solved with non-zero boundary conditions at the same time. Based on Gurtin and Murdoch theory [15], the surface constitutive equation, i.e., the relation between surface stress and surface strain is given by:

$\sigma_{\alpha \beta}^{s}=\tau^{s} \delta_{\alpha \beta}+\left(\tau^{s}+\lambda^{s}\right) \varepsilon^{s}{ }_{v \nu} \delta_{\alpha \beta}+2\left(\mu^{s}-\tau^{s}\right) \varepsilon_{\alpha \beta}^{s}$

where $\sigma_{\alpha \beta}^{s}$ and $\varepsilon_{\alpha \beta}^{s}$ are the surface stress and strain, respectively. $\lambda^{s}$ and $\mu^{s}$ are Lame constants for the surface layer and $\tau^{s}$ is the residual stress on surface layer in the absence of strain.

\subsubsection{Diffusion-induced stresses combined with surface stresses}

After simplification of Eq. (19) by assuming $2 \mu^{s}+\lambda^{s}-\tau^{s}=k^{s}$ and $\tau^{s}=\tau^{0}$, for the planar electrode in the present study, the surface constitutive relationship for an isotropic and homogeneous surface layer in $y$ direction is written as follows:

$\sigma_{y y}^{\text {surf }}=\tau_{\text {surf }}=\tau^{0}+k^{s}\left(\varepsilon_{y y}^{s}-\frac{\Omega c_{s}}{3}\right)$

where $\Omega$ is the partial molar volume of the solute, $c_{s}$ is the concentration for the outer layer, $\varepsilon^{s}{ }_{y y}$

is the stain in $y$ direction for the outer layer, $\tau^{0}$ is the deformation-independent surface tension, and $k^{s}$ is the surface modulus [61,62].

Conventionally, expanding the surface constitutive equation based on elastic strain is the primary approach. 
By using an analogy between thermal and DIS and assuming a homogeneous and isotropic bulk material, the stress-strain relationship in the planar electrodes can be written as follows:

$$
\begin{aligned}
& \varepsilon_{y y}=\frac{1}{E}\left[\sigma_{y y}-v \sigma_{x x}\right]+\frac{1}{3} \Omega c \\
& \varepsilon_{x x}=\frac{1}{E}\left[\sigma_{x x}-v \sigma_{y y}\right]+\frac{1}{3} \Omega c
\end{aligned}
$$

Having assumed that the concentration distribution occurs at a much slower pace, the equilibrium equation in each step in $y$ direction can be expressed as the following equation:

$$
\int_{-h}^{+h} \sigma_{y y} d x+2 \tau_{\text {surf }}=0
$$

Through the use of the compatibility equation and symmetry, we may write:

$$
\frac{\partial^{2} \varepsilon_{y y}}{\partial x^{2}}=0
$$

where $E$ is Young modulus, $v$ is Poisson ratio, $c$ is molar concentration, $\varepsilon_{x x}$ and $\varepsilon_{y y}$ are strains in the $x$ and $y$ directions and $\sigma_{y y}$ is stress in the $y$ direction.

After combining Eqs. (20)-(24), the stress distribution caused by deintercalation and surface stress is obtained as follows:

$$
\tilde{\sigma}_{D}=\tilde{\sigma}_{y y}=\frac{1}{3}\left[\frac{1}{1+\tilde{\lambda}(1-v) k^{*}} \tilde{c}_{a v g}-\tilde{c}+\frac{\tilde{\lambda}(1-v) k^{*}}{1+\tilde{\lambda}(1-v) k^{*}} \tilde{c_{s}}\right]-\frac{\tau^{*} \tilde{\lambda}}{1+\tilde{\lambda}(1-v) k^{*}}
$$

where $\tilde{\lambda}=\frac{\lambda}{h}$ is dimensionless phase boundary thickness parameter, $c_{a v g}=\frac{1}{2 h} \int_{-h}^{+h} c(x) d x$ is the average concentration, $B=\frac{E}{1-v}$ is the biaxial elastic modulus, $\tau^{*}=\frac{\tau_{0}}{B \Omega c_{\max } \lambda}$ is the 
dimensionless surface stress parameter, $k^{*}=\frac{k^{s}}{E \lambda}$ is the dimensionless surface modulus parameter, and $\tilde{\sigma}_{y y}=\frac{\sigma_{D}(1-v)}{E \Omega c_{\max }}$ is the dimensionless diffusion-induced stress.

It is assumed that $\tau_{0}=0.1 \frac{N}{m}$ and $k^{s}= \pm 10 \frac{N}{m}[32,33,56,61,63]$ and the other parameters are set as given in Table 1 for $\mathrm{LiMn}_{2} \mathrm{O}_{4}$.

Table 1: Mechanical and chemical properties of $\mathrm{LiMn}_{2} \mathrm{O}_{4}$

\begin{tabular}{|c|c|c|c|c|}
\hline$E(\mathrm{Gpa})$ & $v$ & $\Omega\left(\frac{\mathrm{m}^{3}}{\mathrm{~mol}}\right)$ & $c_{\max }\left(\frac{\mathrm{mol}}{\mathrm{m}^{3}}\right)$ & $\lambda(\mathrm{nm})$ \\
\hline $87[67]$ & $0.26[68]$ & $3.26 \times 10^{-6}[69]$ & $2.37 \times 10^{4}[70]$ & $1[51]$ \\
\hline
\end{tabular}

Based on the values of the above parameters, the dimensionless parameters are calculated as $k^{s}=0,-0.1,+0.1$ and $\tau^{*}=0,0.05,0.1$ in this research.

As an alternative approach, which is based on total strain, the outer surface constitutive equation is expressed as follows:

$$
\tau_{\text {surf }}=\tau^{0}+k^{s} \varepsilon_{y y}
$$

Therefore, the diffusion-induced stress in the presence of surface stress is obtained as:

$$
\tilde{\sigma}_{D}=\tilde{\sigma}_{y y}=\frac{1}{3}\left[\frac{1}{1+\tilde{\lambda}(1-v) k^{*}} \tilde{c}_{a v g}-\tilde{c}\right]-\frac{\tau^{*} \tilde{\lambda}}{1+\tilde{\lambda}(1-v) k^{*}}
$$

It is worth noting that Eq. (20) was used in Refs. [30-32], on the other hand, Eq. (26) was used in other Refs. [29,33]. It should be noted that Eq. (20) appears to be more accurate than Eq. (26), since in Eq. (20), $\frac{1}{3} \Omega c$ is diffracted from the total strain in the calculation of elastic strains, which 
is similar to the bulk constitutive equations (Eqs. (21) and (22)). Therefore, on the right-hand side of the Eq. (20), $\frac{1}{3} \Omega c_{s}$ is diffracted from total strain of the outer layer while elastic strains of the outer layer are considered in the equation.

\subsection{Fracture mechanics problem}

Fracture mechanics theory is able to predict the failure mode accurately for structures when defects such as cracks exist beforehand in the structures [71]. Various analytical and numerical methods exist for solving fracture mechanics problems [71]. Weight function method, as a widely used analytical method is employed in this study due to its efficiency and low computational cost.

\subsubsection{Weight function method}

The weight function method introduced by Buckner [72] and Rice [70] is an efficient method for the calculation of stress intensity factor especially when other methods such as energy methods are not available or time-consuming to be employed. In addition, for the specific problem discussed here, the stresses vary over time and position; therefore, this method is utilized in this research. Following this approach, the stress intensity factor is calculated using Eq. (28):

$$
K_{I}=\int_{0}^{a} w(x) \sigma_{x} d x
$$

where $\sigma_{x}$ represents the distribution of the normal traction along the crack face on the uncracked body when it is subjected to the loading system, which can be calculated by Eq. (25) in this study, $w(x)$ is the SIF induced at the tip of a crack when crack face is opened up by two opposite unit point loads acting normal to the crack face at distance $x$ from the centerline of the crack. This parameter depends only on the geometry of the particle and is independent of loading. 
For the edge crack problem, the integral is expressed in the following form:

$$
K_{I}=\int_{h-a}^{h} w_{e}(x) \sigma_{y y} d x
$$

Tada [74] gives $w_{e}(x)$ for this problem as follows:

$$
w_{e}(x)=\frac{2}{\sqrt{\pi a}} \frac{G_{e}\left(\frac{h-x}{a}, \frac{a}{2 h}\right)}{\left(1-\frac{a}{2 h}\right)^{\frac{3}{2}} \sqrt{\left(1-\left(\frac{h-x}{a}\right)^{2}\right)}}
$$

where

$$
\begin{aligned}
& G_{e}(\xi, \eta)=g_{1}(\eta)+g_{2}(\eta) \xi+g_{3}(\eta) \xi^{2}+g_{4}(\eta) \xi^{3} \\
& g_{1}(\eta)=0.46+3.06(\eta)+0.84(1-\eta)^{5}+0.66(\eta)^{2}(1-\eta)^{2} \\
& g_{2}(\eta)=-3.52(\eta)^{2} \\
& g_{3}(\eta)=6.17-28.22(\eta)+34.54(\eta)^{2}-14.39(\eta)^{3}-(1-\eta)^{\frac{3}{2}}-5.88(1-\eta)^{5}-2.64(\eta)^{2}(1-\eta)^{2}
\end{aligned}
$$

$g_{4}(\eta)=-6.63+25.16(\eta)-31.04(\eta)^{2}+14.41(\eta)^{3}+2(1-\eta)^{\frac{3}{2}}+5.04(1-\eta)^{5}+1.98(\eta)^{2}(1-\eta)^{2}$

Thereafter, Eqs. (31)-(35) are substituted into Eq. (30) and then into Eq. (29). Furthermore, by substituting Eq. (25) into Eq. (29), the resulting dimensionless stress intensity factor is obtained as follows:

$$
\tilde{K}_{I}=\int_{h-\tilde{a}}^{\tilde{h}} \frac{2}{\sqrt{\pi \tilde{a}}} \tilde{G}_{e}\left(-\tau^{*}\left(\frac{1}{k^{*}+\frac{1}{\tilde{\lambda}}}\right)+\frac{\tilde{c}_{s}}{3}\left(\frac{k^{*}}{k^{*}+\frac{1}{\tilde{\lambda}}}\right)+\left(\frac{1}{3 \lambda_{c}\left(k^{*}+\frac{1}{\tilde{\lambda}}\right)}\right) \tilde{c}_{a v g}-\frac{\tilde{c}}{3}\right) d \tilde{x}
$$


Obviously, the integral in Eq. (36) has a singular point at the lower bound. Therefore, it is necessary to implement an efficient numerical integration method to eliminate the singular point. Accordingly, the method employed by Fett [75] is used in which the integral is divided into two parts.

\section{Results and Discussion}

In this section, the concentration, diffusion-induced stress and stress intensity factor in the presence of surface stress are determined. The correlation between the phase-separation parameters and surface stress parameters is clarified in order to understand which one is the dominant factor for different cases.

\subsection{Concentration profile}

In this section, the effect of evolution of concentration profiles for classical and phase-separating diffusion over time is discussed. In addition, the effect of phase boundary thickness parameter is investigated for phase-separating diffusion.

\subsubsection{Classical diffusion}

After solving the classical diffusion equation (Fick's law) according to finite difference method, the concentration profile is plotted for deintercalation process for different values of dimensionless time in Fig. 2. As shown in the figure, the dimensionless concentration value starts from unity and gradually decreases until the process is completed in which the concentration value reaches zero. 


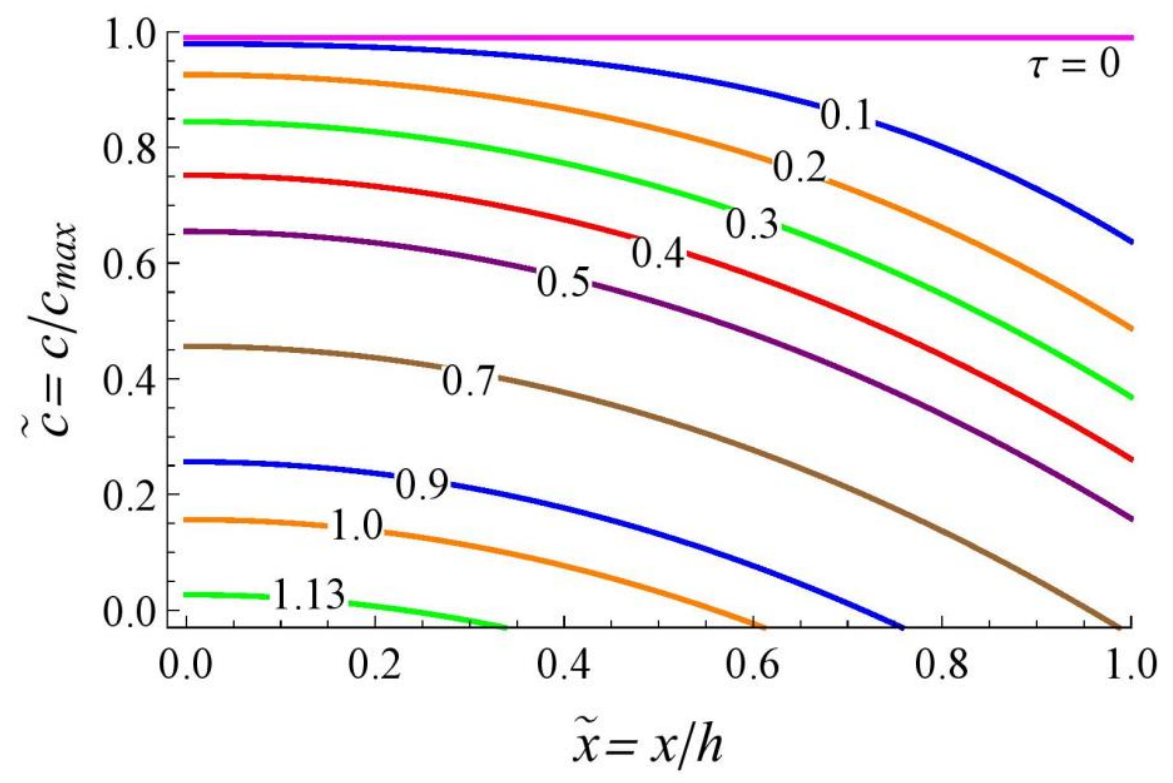

Fig. 2: Concentration profile for deintercalation process

\subsubsection{Phase-separating diffusion}

Fig. 3 shows variation of the concentration profile with time for different values of the dimensionless parameter $\frac{\lambda}{h}$ during the deintercalation. The three possible values of $\frac{\lambda}{h}$ were chosen such that the effect of $\lambda$ and transition zone would be visible. It is seen that due to the deintercalation, in the first step, the dimensionless concentration is equal to unity, and as the process proceeds, the concentration reduces until it reaches zero at the end of the process. With reducing the parameter $\lambda$, the phase-separation phenomenon becomes more influential, so that a sharper concentration profile is observed. For example, if $h=50 \lambda$ (Fig. 3(c)), the phaseseparation phenomenon is clearly seen in the width of the electrode. In other words, the two different zones with different concentrations would emerge. These concentrations are almost the minimum values in the homogeneous energy diagram. With decreasing $\frac{h}{\lambda}$, e.g., $h=2 \lambda$ or $h=5 \lambda$ (Fig. 3(b)), the difference between the zones is observed to reduce so a steep slope is not seen as 
shown in Fig. 3(a). This is due to the fact that in phase-field model $\lambda$ (thickness) is a pivotal factor that controls and determines the phase boundary thickness. Therefore, when $\frac{h}{\lambda}$ decreases, in which $h$ and $\lambda$ are comparable with each other, the phase boundary thickness covers the whole width of the electrode and distinguishable phases do not emerge, which is known as "nanoconfinement". As shown in Fig. 3(c), in the early stages of deintercalation process, the concentrations in the vicinity of the center of the electrode are in the same range contrary to further electrodes. The reason is that in the early stages in deintercalation process, most of the lithium ions moves apart from the surface of the particle, while most of the ions locating at the center of the particle remain at their initial position.

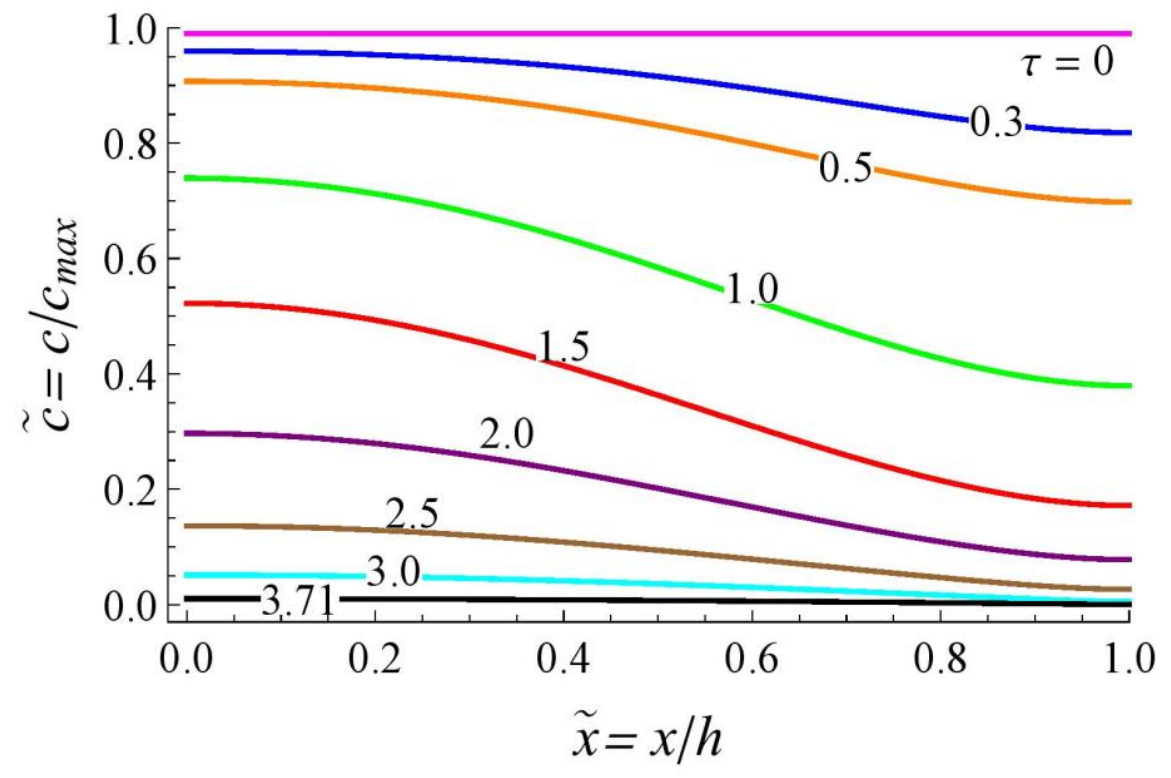

(a) 


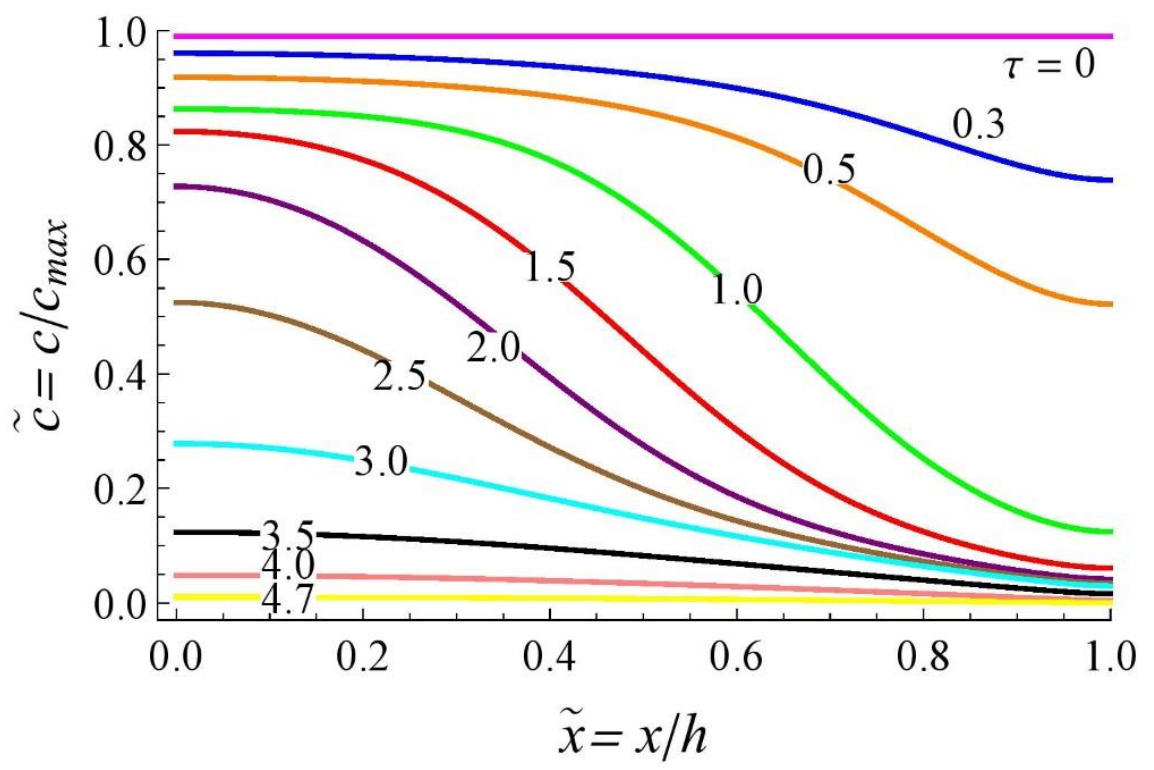

(b)

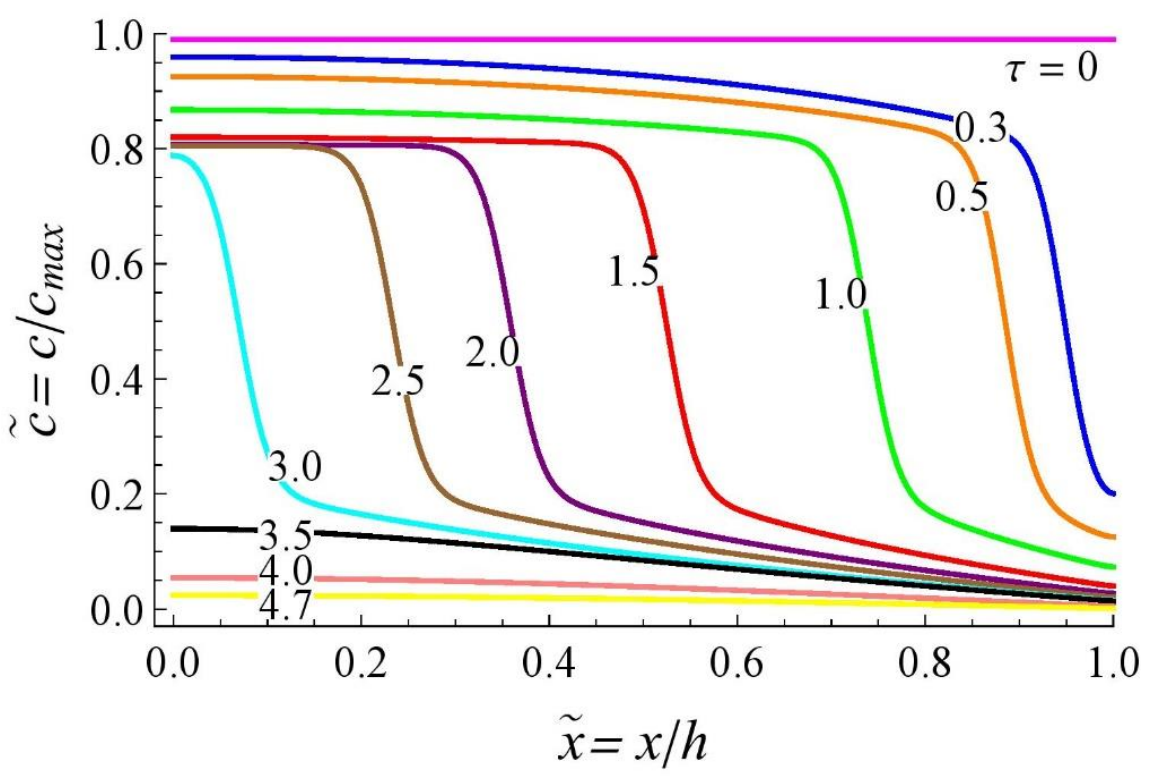

(c)

Fig. 3: Evolution of the concentration profile during deintercalation $\left(\tilde{j}_{0}=1, \tilde{\omega}=2.31\right)$ predicted by the phase-field model (a) $\frac{\lambda}{h}=0.5$ (b) $\frac{\lambda}{h}=0.2$ (c) $\frac{\lambda}{h}=0.02$ 
As shown in the figure, the slope of the concentration profiles are steeper for phase-separation diffusion compared to the concentration profile for classical diffusion, which is attributed to the phase-separation phenomenon. Additionally, the concentration profile for classical diffusion is more affected by the surface flux compared to the phase-separation concentration profiles. The results of Figs. 2 and 3 are in good agreement with the reported results in Refs. [27] and [42] for classical and phase-separating diffusion.

\subsection{Stress profile}

In this section, the effects of dimensionless surface stress, surface modulus, and time, and two different approaches for surface constitutive equation are discussed based on elastic strain or total strain on the stress profile for classical and phase-separating diffusion. Additionally, the effect of phase boundary thickness parameter $\left(\frac{\lambda}{h}\right)$ is addressed in the phase-separating diffusion section.

\subsubsection{Classical diffusion}

The stress profile for deintercalation process is illustrated in Fig. 4 for $\tilde{j}_{0}=1, k^{*}=0$ for four different values of dimensionless time during the process. It is noted that, two possible values for dimensionless surface stress parameter $\tau^{*}=0.05,0.1$ are also chosen. As it is clearly seen, with increasing the $\tau^{*}$, the tensile stresses in the width of the particle decrease and the compression stresses increase. Therefore, a drop in the diagrams in each dimensionless time is visible. It is due to the fact that by increasing the $\tau^{*}$, the tensile stresses in the outer layer increase according to Eq. (20). Thus, in order to satisfy the equilibrium condition in the whole width of the electrode (zero axial load), the diffusion-induced stresses in the width of the electrode decline while the compressive stresses escalate. 


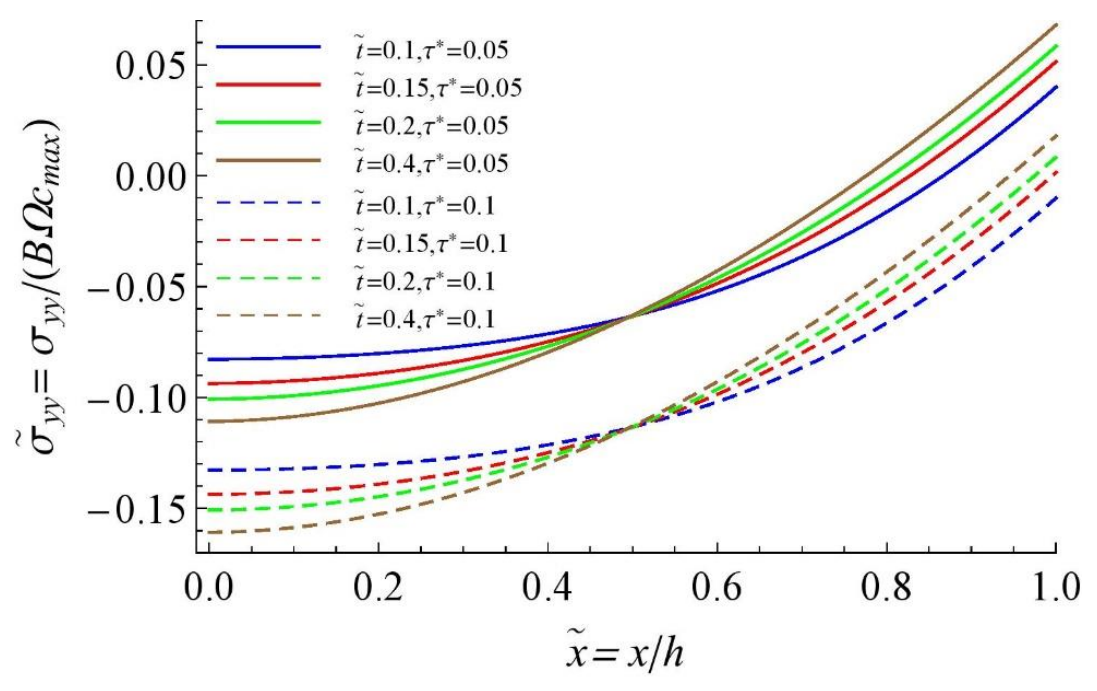

Fig. 4: Evolution of the stress profile during deintercalation predicted by the classical diffusion model for $\tilde{j}_{0}=1$ and $k^{*}=0$

Fig. 5 shows the stress profile for deintercalation process for $\tilde{j}_{0}=1, \tau^{*}=0.05$ for four different values of dimensionless time during the process. Two possible values for dimensionless surface modulus parameter $k^{*}=-0.1,0.1$ are also chosen in order to consider the possible effects of the dimensionless surface modulus. As can be seen, when the elastics strain in the deintercalation process is positive, i.e., when $k^{*}$ is positive, the surface stress in the outer layer (Eq. (20)) is tensile. Therefore, due to the equilibrium condition (see Eq. (23)), the diffusion-induced stresses in the width of the particle reduce and become more compressive. The results of Figs. 4 and 5 are in good agreement with the reported outcomes in Refs. [30-33] in which the positive surface stress parameters reduce the stresses caused by classical diffusion. 


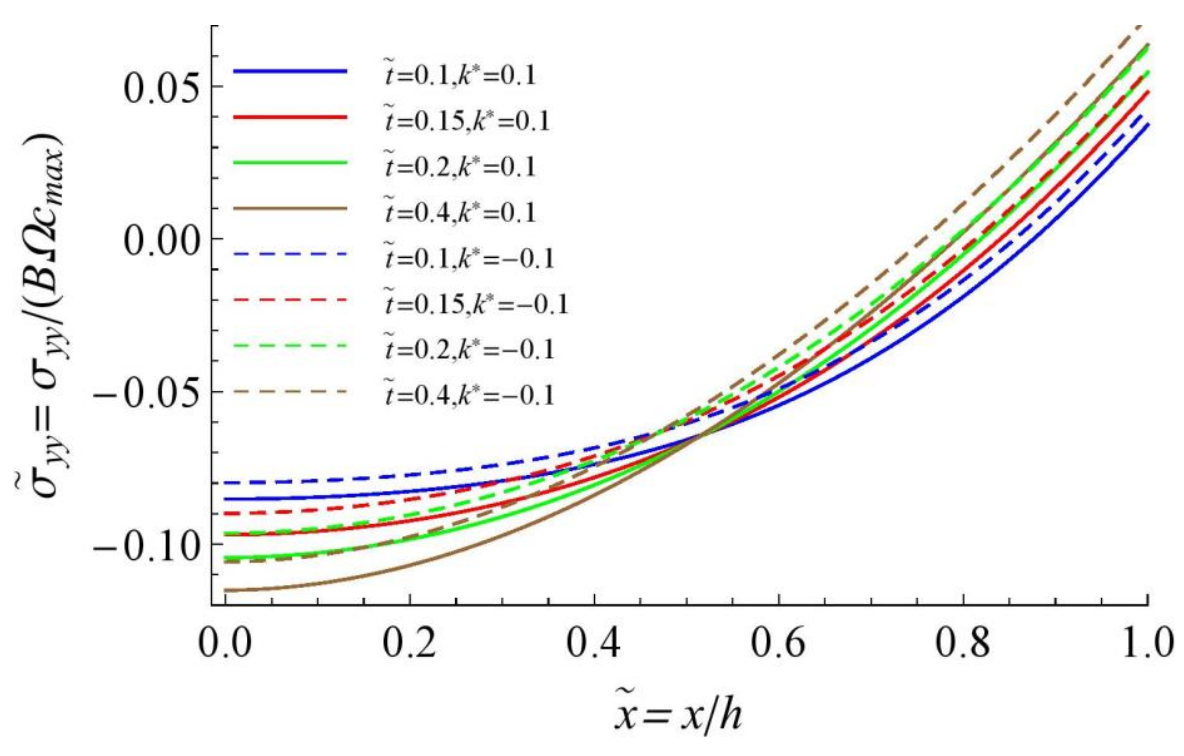

Fig. 5: Evolution of the stress profile during deintercalation predicted by the classical diffusion model for $\tilde{j}_{0}=1$ and $\tau^{*}=0.05$

The comparison between two available alternatives provided for surface constitutive equation based on elastic strain (Eq. (20)) and total strain (Eq. (26)) is illustrated in Fig. 6 for $\tau^{*}=0.1$, $k^{*}=0.1$ and $\tilde{j}_{0}=1$. Evidently, the elastic strain values are observed to be higher than the corresponding values of total strain so that less compressive stresses are expected. In addition, the differences between the two alternative assumptions reduce over the stress profile with time. This can be justified because with regard to deintercalation process and the formulation of elastic strain, the right-hand side of the Eq. (20) reduce higher compared to the right-hand side of the Eq. (26), which is based on the total strain. Therefore, concerning the formulation of elastic strain, the tensile stresses in the outer layer and compressive stresses in the width of the electrode are smaller. Additionally, because of the deintercalation process, the differences between the right-hand side of the Eq. (20) and Eq. (26) decrease over time, which is accompanied by a decrease in concentration. 


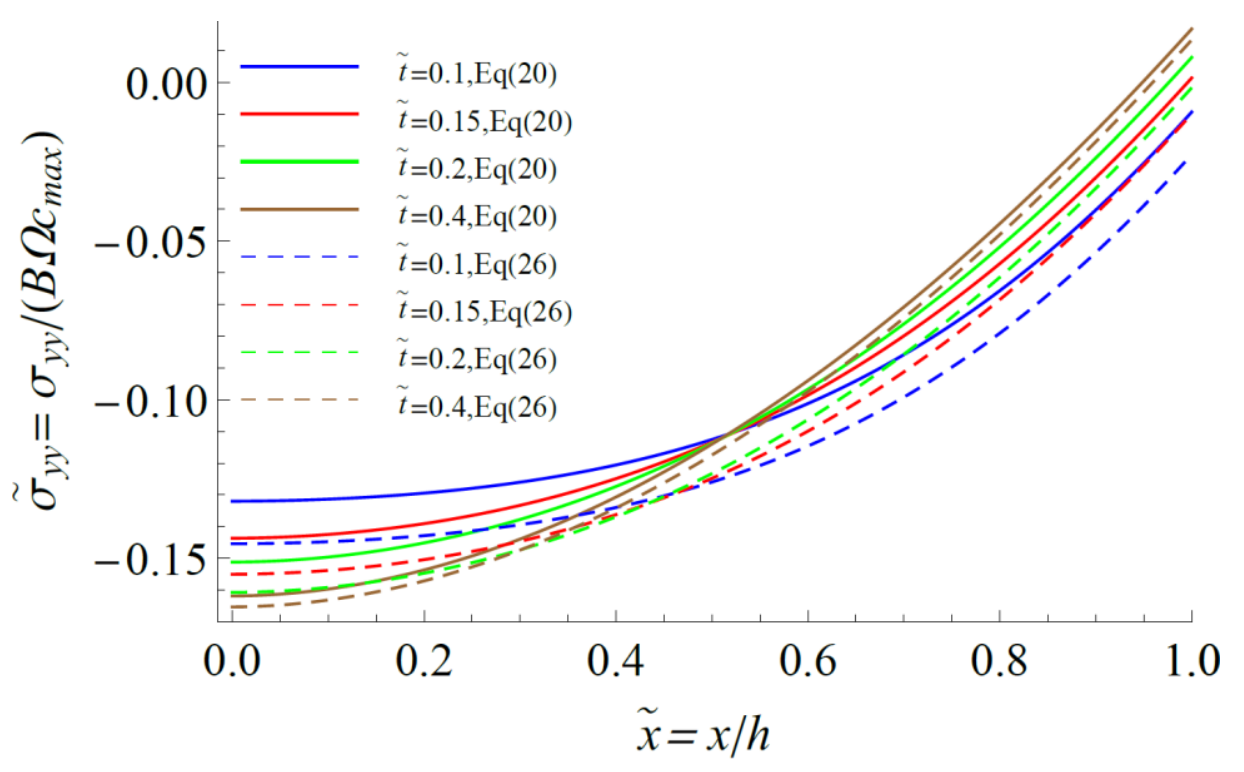

Fig. 6: Evolution of the stress profile during deintercalation predicted by the classical diffusion

$$
\text { model for } \tau^{*}=0.1, k^{*}=0.1 \text { and } \tilde{j}_{0}=1
$$

\subsubsection{Phase-separating diffusion}

The corresponding stress profiles for the plotted concentration profiles is illustrated in Fig. 7 for $\tilde{j}_{0}=1, \tilde{\omega}=2.31$ and different values of $\tau^{*}$ in addition to three dimensionless time values during the deintercalation. It is clear that, similar to the concentration profile, the stress profile is sharpened when the parameter $\lambda$ reduces. As the process proceeds, the positive stresses are maximized, with regard to the time for the abrupt drops in concentration profile, and then are reduced to zero at the end of the process.

With the increment of $\tau^{*}$, the tensile stresses in each time step decrease, whereas the compression stresses increase, and thus the stress profile declines when $\tau^{*}$ raises. Similar to the classical diffusion, tensile surface stresses would exist on the outer layer of the particle. Therefore, the 
equilibrium condition should be satisfied in such a way that the diffusion-induced stresses reduce in the width of the electrode while taking negative values.

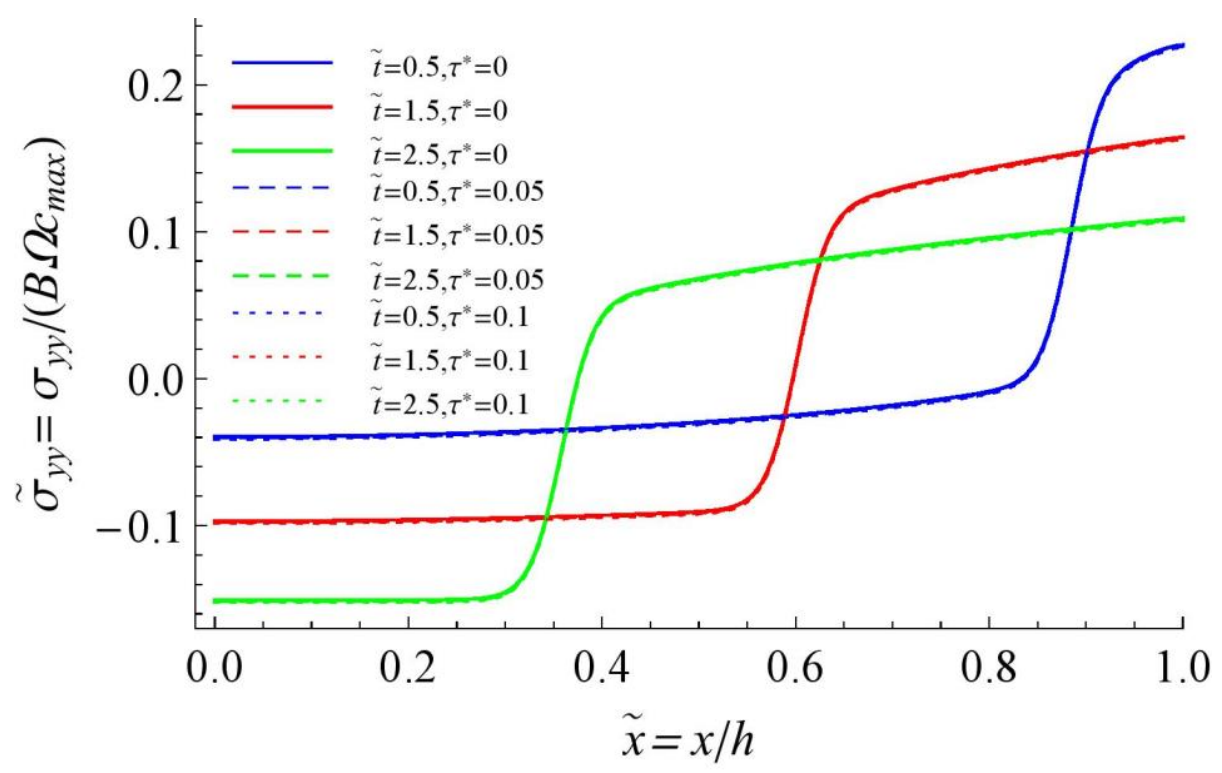

(a)

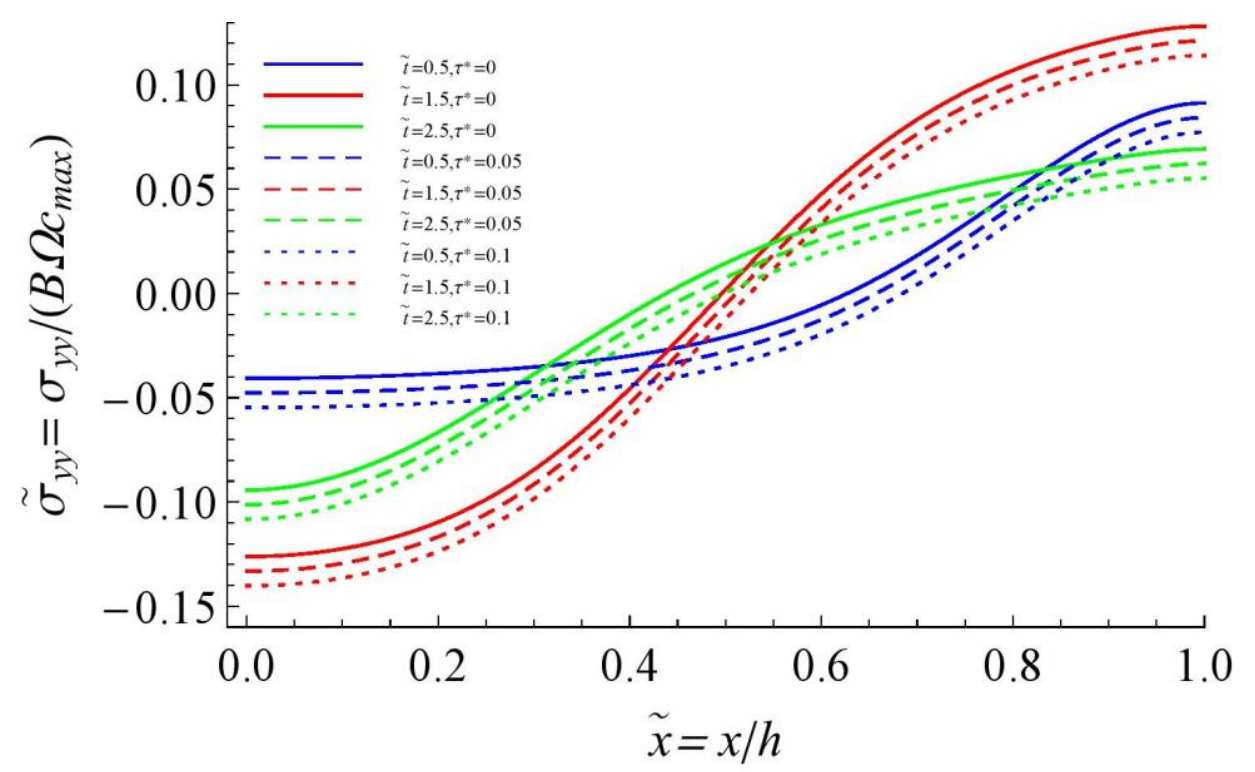

(b) 


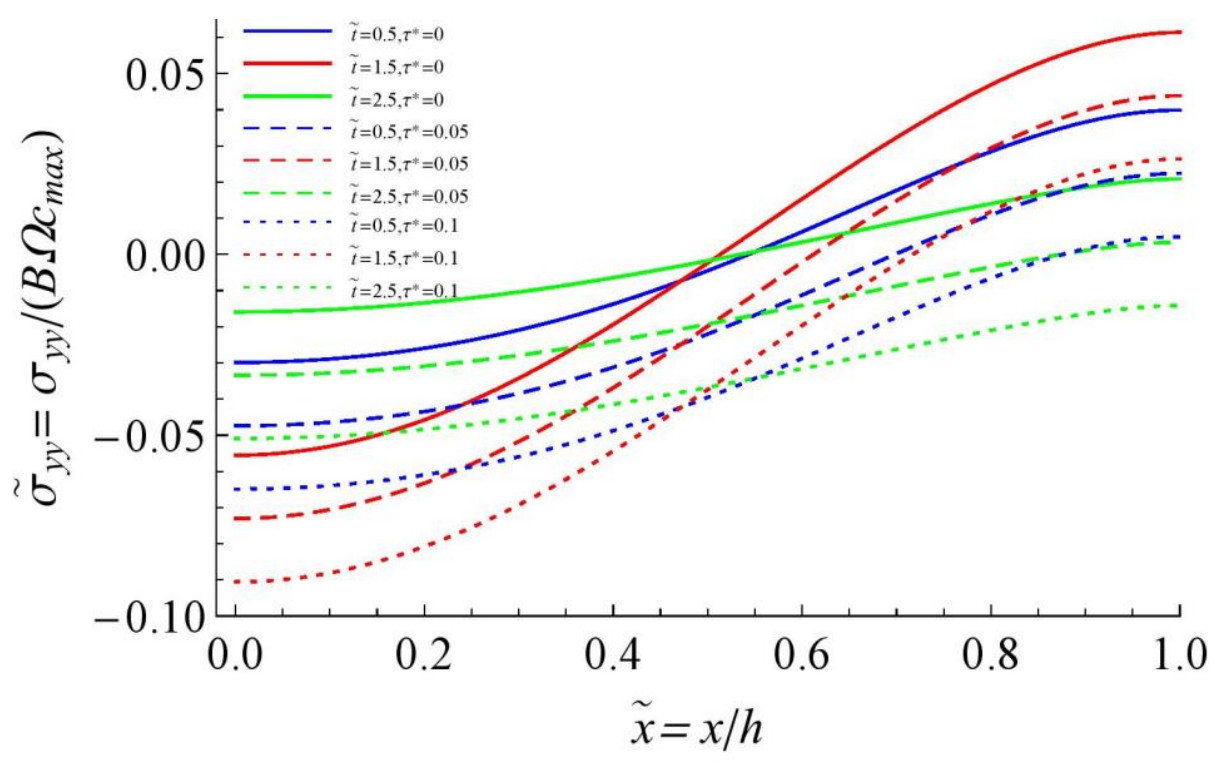

(c)

Fig. 7: Evolution of the stress profile during deintercalation for $\tilde{j}_{0}=1, \tilde{\omega}=2.31$ and three

values of dimensionless surface stress parameter and time predicted by the phase-field model (a)

$$
\frac{\lambda}{h}=0.02 \text { (b) } \frac{\lambda}{h}=0.2 \text { (c) } \frac{\lambda}{h}=0.5
$$

Figure 8 illustrates stress profiles associated with the relative concentration profiles for $\tilde{j}_{0}=1$, $\tilde{\omega}=2.31$, different possible values of $k^{*}$ and three dimensionless times during the deintercalation. A similar trend as observed in Fig. 7 is seen here except the effect of $k^{*}$ which is less pronounced than $\tau^{*}$, consisting with Eq. (26) for the same dimensionless surface stress in each time. Similar to the classical diffusion, when $k^{*}$ is positive, tensile surface stress is created in the outer layer, compressive diffusion-induced stresses are created in the width of the particle to achieve equilibrium. The comparison between Fig. 7 and Fig. 8 indicates that although in Fig. 7 the differences between the diagrams for the same $\tau^{*}$ remain constant with time, in Fig. 8 the difference between the diagrams for the same $k^{*}$ increases up to the maximum time then starting 
to decline until the process is over. The reason is that the term $\tau^{*}$ in Eq. (26) is independent of time and concentration, while $k^{*}$ is time dependent.

The most important criteria of Fig. 7 and Fig. 8 is that when $\lambda$ increases, the effects of $\tau^{*}$ and $k^{*}$ become more evident, and the differences between the diagrams increase for different dimensionless surface stresses at the same time. This can be supported by Eq. (26) and shows that the effects of surface mechanics parameters are dependent on phase boundary thickness.

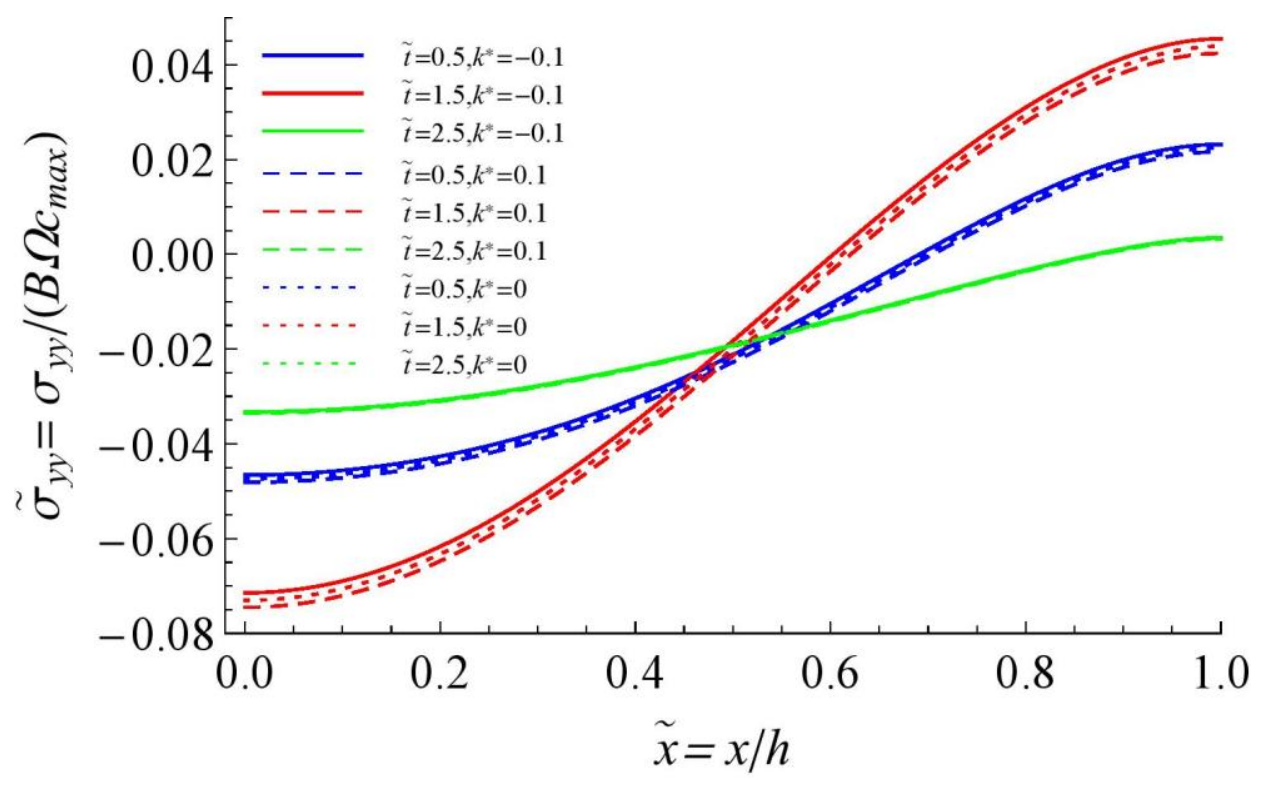

(a) 


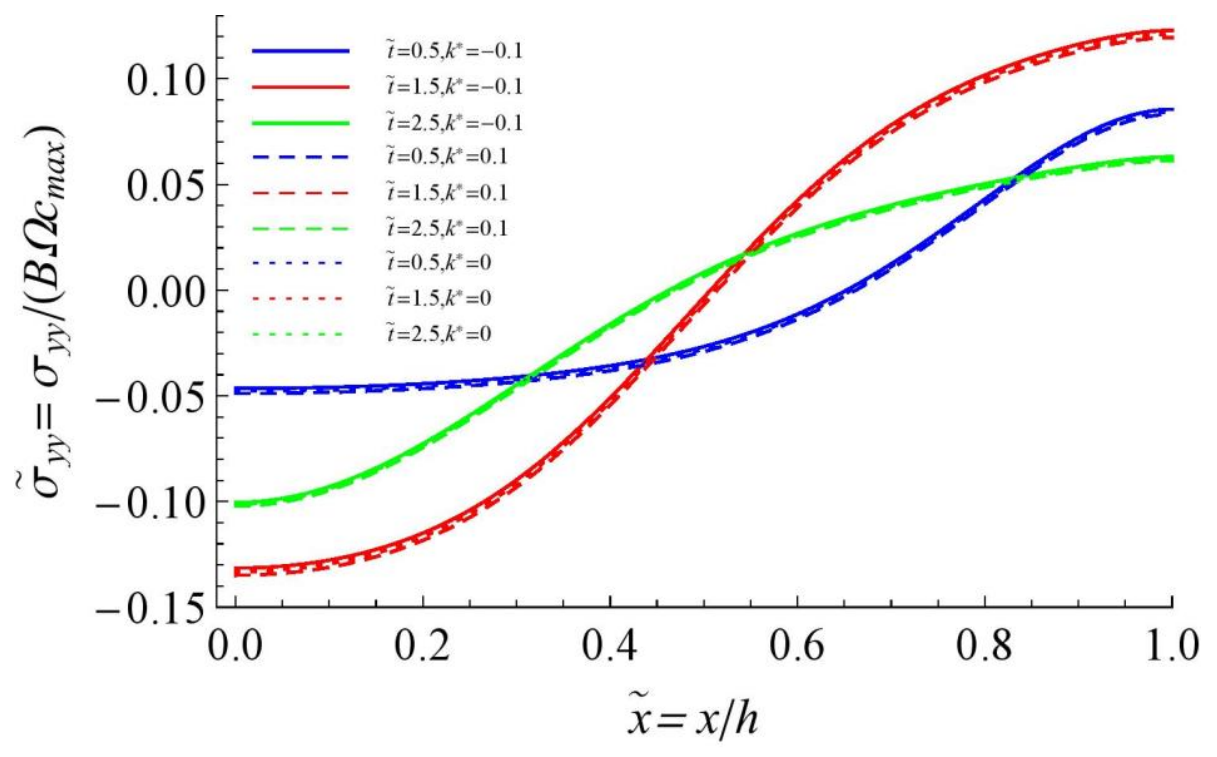

(b)

Fig. 8: Evolution of the stress profile during deintercalation for $\tilde{j}_{0}=1, \tilde{\omega}=2.31$ and three

values of dimensionless surface stress and time predicted by the phase-field model (a) $\frac{\lambda}{h}=0.5$ (b)

$$
\frac{\lambda}{h}=0.2
$$

Figure 9 shows the maximum stress calculated with the help of Eq. (26) versus $\frac{h}{\lambda}$ for $\tilde{j}_{0}=1$, $\tilde{\omega}=2.31$ and different values of $k^{*}$ and $\tau^{*}$. Obviously, with the increment of $\frac{h}{\lambda}$, the maximum stress increases as well, which can be attributed to the phase-separation phenomenon.

For small values of $\frac{h}{\lambda}$ the slope of the diagrams are much steeper than the large values of $\frac{h}{\lambda}$ and for higher values of $\frac{h}{\lambda}$, the curve converges to the values predicted by analytical method, which is calculated based on core-shell model [43]. 
As discussed before, for higher values of $\tau^{*}$, the maximum stress reduces for each $\frac{h}{\lambda}$ and for positive values of $k^{*}$, the maximum stress would drop for each $\frac{h}{\lambda}$ compared to zero and negative values of $k^{*}$. These maximum stresses in Fig. 9 for each $\frac{h}{\lambda}$ are associated with the end of stress profiles among all the times in the deintercalation process which has the highest value (Fig. 7 and Fig. 8).

The results of Figs. 7 and 8 are similar to those of Ref. [42] when surface stress parameters are neglected $\left(\tau^{*}=0, k^{*}=0\right)$.

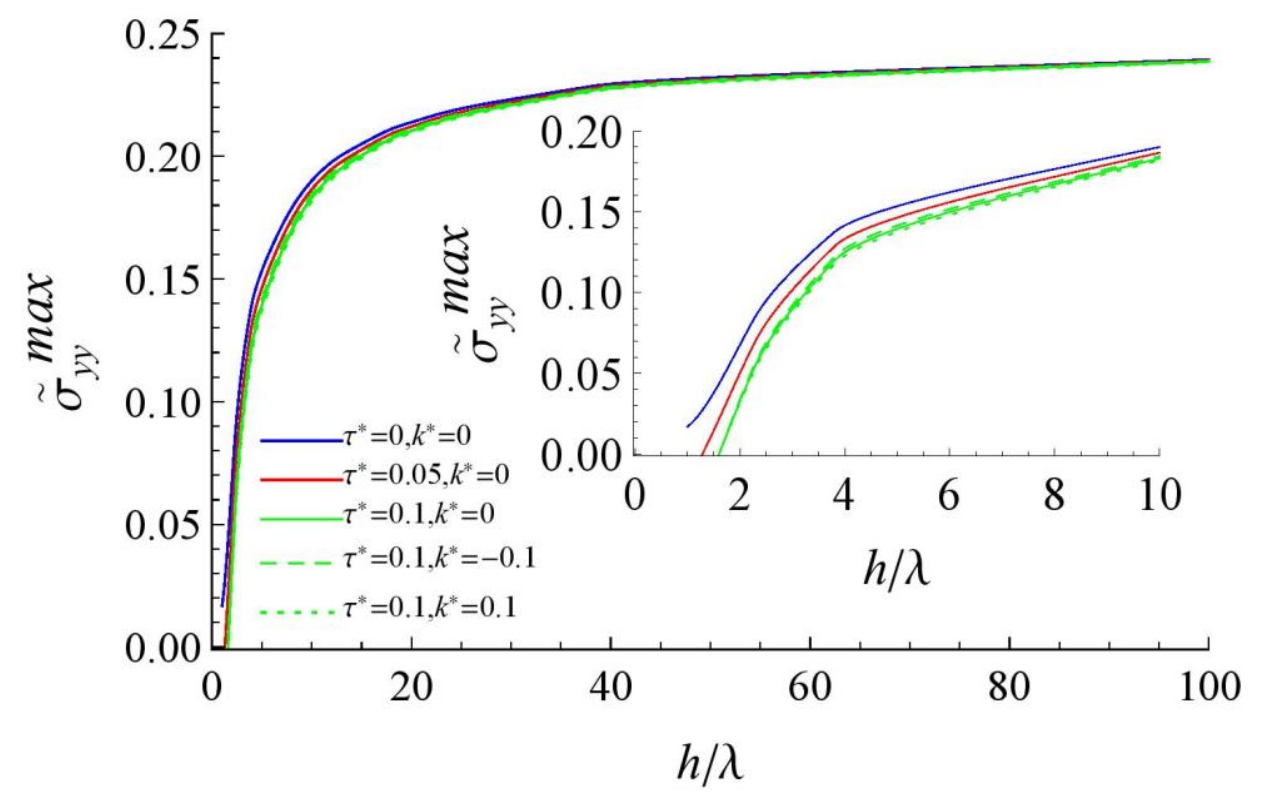

Fig. 9: Maximum stress in the width of planar electrode versus phase boundary thickness parameter for $\tilde{j}_{0}=1, \tilde{\omega}=2.31$ and different possible values of dimensionless surface stress and surface modulus 
The comparison between two alternative methods for surface constitutive equation based on elastic strain (Eq. (20)) and total strain (Eq. (26)) is shown in Fig. 10 and Fig. 11 for $\tilde{j}_{0}=1$ and $\tilde{\omega}=2.31$ $h=2 \lambda, k^{*}=-0.1,0.1$ and $\tau^{*}=0.05$. Similar to the classical diffusion, when $k^{*}=0.1$, the stress values concluded by Eq. (20) are higher than the values estimated by the formulation of total strain so less compressive stresses are created. Additionally, the differences between these two formulations reduces in the stress profile with time. However, for $k^{*}=-0.1$ the trend is reversed. The stress values in Fig. 10 and Fig. 11 are higher than those in Fig. 6 due to the phase-separation phenomenon, causing steeper slopes.

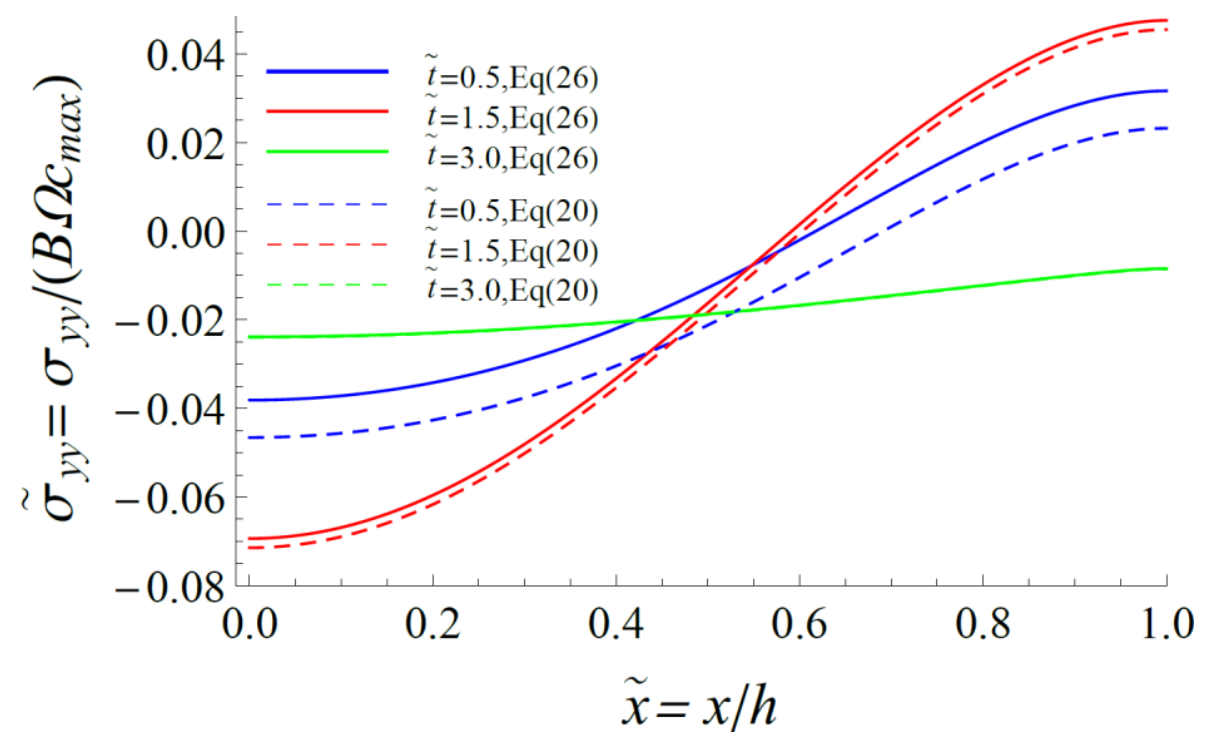

Fig. 10: Evolution of the stress profile during deintercalation predicted by the phase-field model for $\tilde{j}_{0}=1, \tilde{\omega}=2.31, h=2 \lambda, k^{*}=-0.1$ and $\tau^{*}=0.05$ 


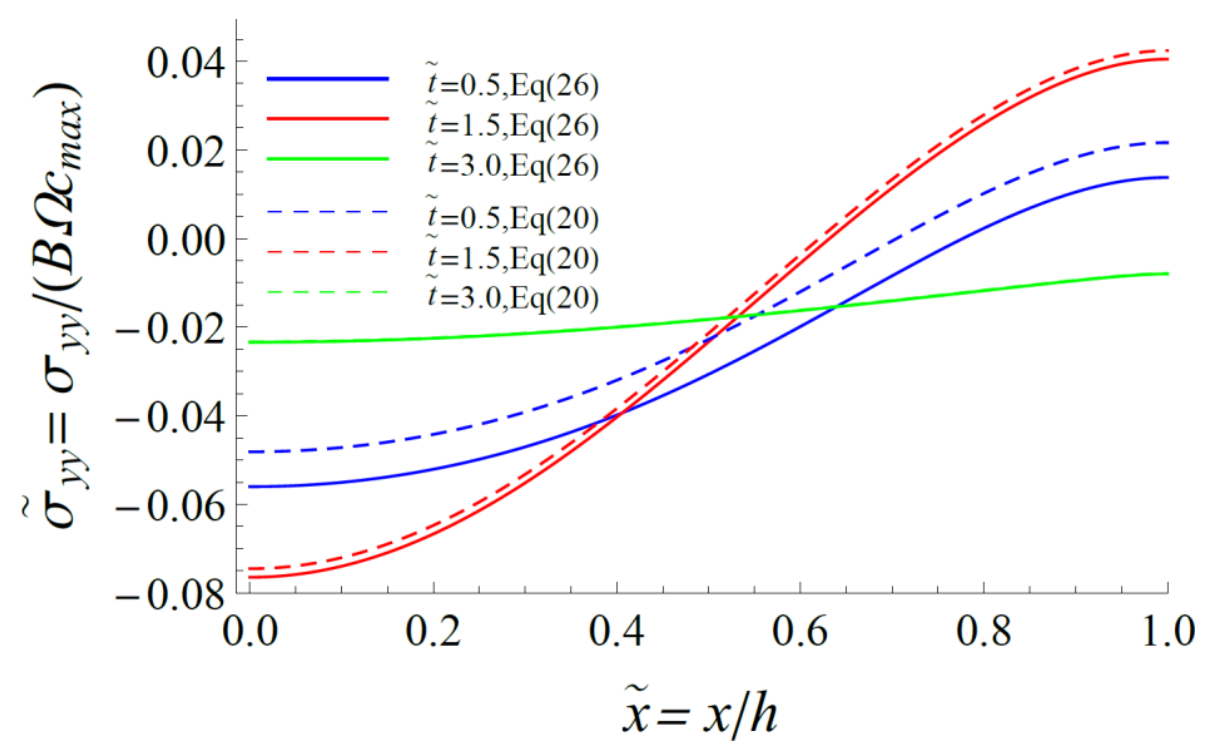

Fig. 11: Evolution of the stress profile during deintercalation predicted by the phase-field model

$$
\text { for } \tilde{j}_{0}=1, \tilde{\omega}=2.31, h=2 \lambda, k^{*}=0.1 \text { and } \tau^{*}=0.05
$$

\subsection{Stress intensity factor}

Figure 12 shows the stress intensity factor versus crack length for three different values of $\tau^{*}$ and dimensionless time $\tilde{t}$ for $\tilde{\lambda}=0.1, k^{*}=0.1, \tilde{j}_{0}=1$ and $\tilde{\omega}=2.31$, where the second value for a dimensionless time corresponds to the time when the maximum stress intensity factor occurs. As shown in the figure, in all cases, whether the surface stress exists or not, the stress intensity factor increases and after being maximized in the second time, it reaches zero. The stability of crack propagation could be understood by stress intensity factor derivative to crack length, i.e., the graph slope. The maximum value of stress intensity factor decreases when the effect of surface stress effect is intensified, and the positive slope, which represents the instability of crack growth occurs in a shorter length. It underscores that, in the presence of surface stress, the crack instability growth is more likely to occur compared to the case where the surface stress is ignored in the particle. 


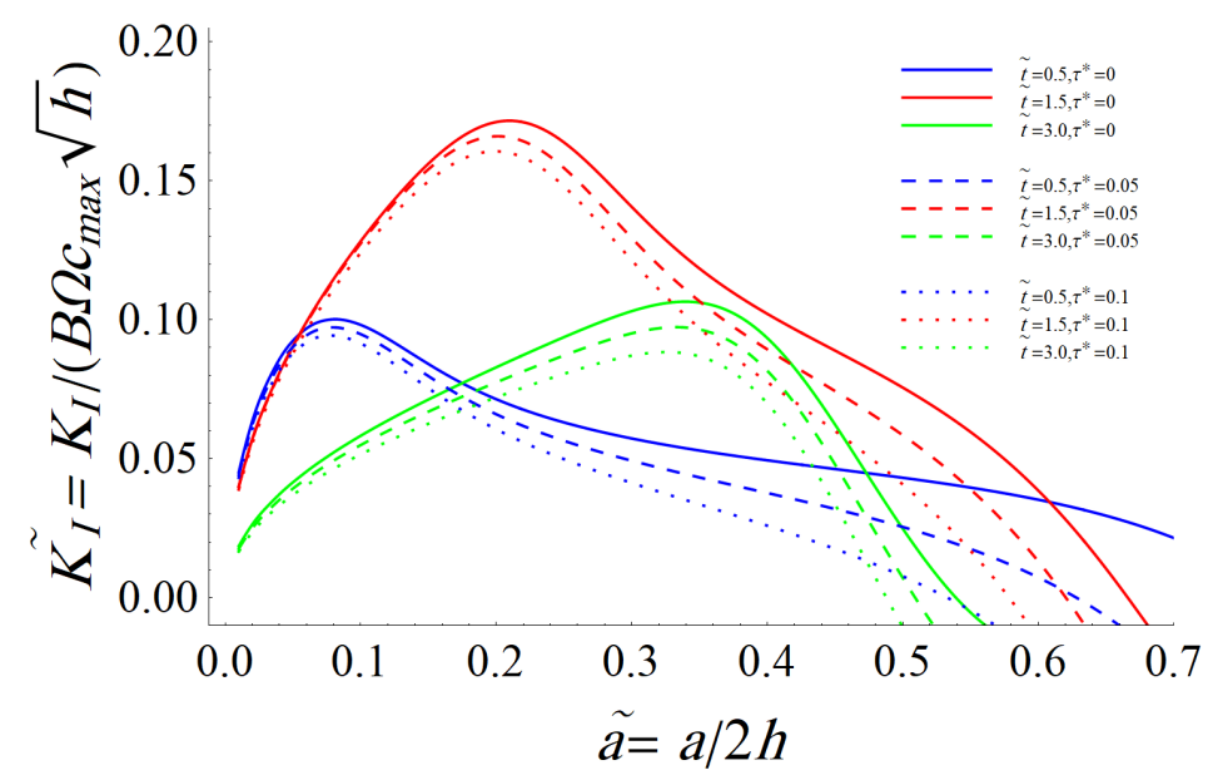

Fig. 12: Stress intensity factor versus crack length for three different possible values of dimensionless surface stress and time for $\tilde{\lambda}=0.1, k^{*}=0.1, \tilde{j}_{0}=1$ and $\tilde{\omega}=2.31$,

Fig. 13 illustrates the maximum stress intensity factors versus phase boundary thickness for $\tilde{j}_{0}=1$ , $\tilde{\omega}=2.31$ and different values of $\tau^{*}$ and $k^{*}$. A similar trend observed in Fig. 5 is dominant here because of the fact that the stress intensity factors is in direct correlation with the stresses according to Eq. (36). 


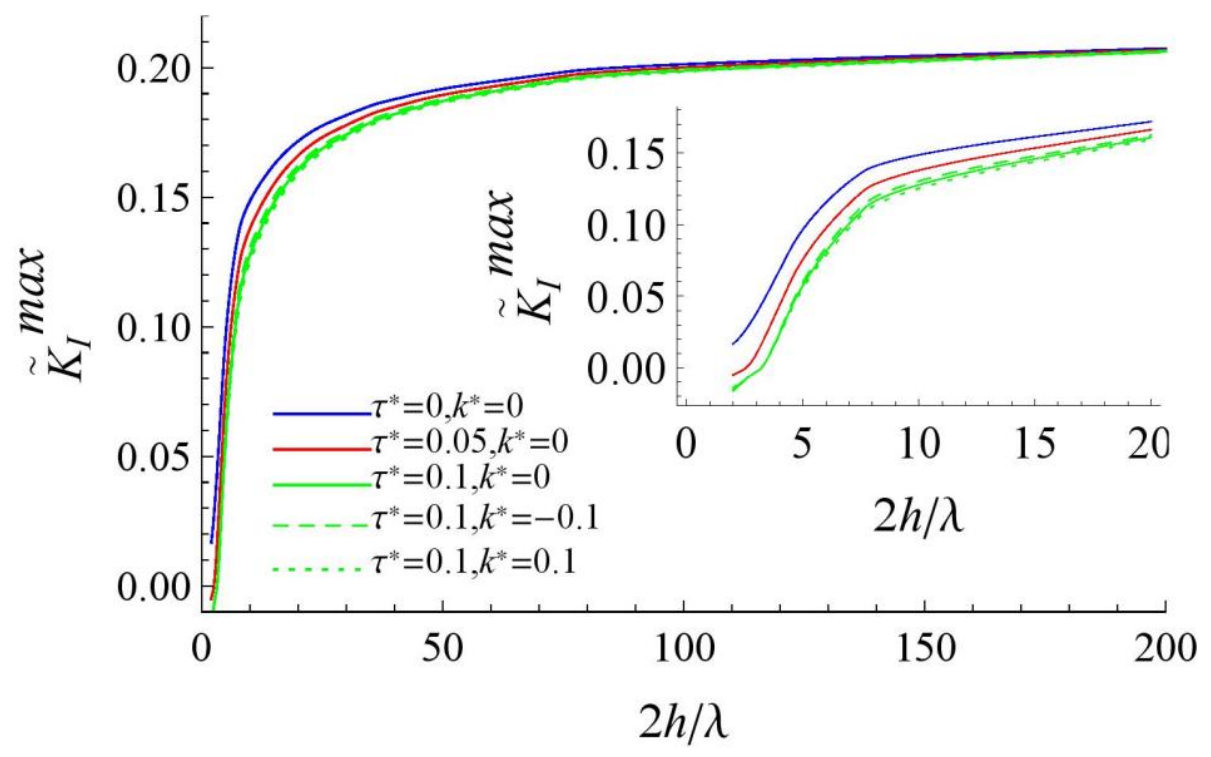

Fig. 13: Maximum stress intensity factor versus phase boundary thickness for $\tilde{j}_{0}=1, \tilde{\omega}=2.31$ and different possible values of dimensionless surface stress and surface modulus values

Figure 14 shows the maximum stress intensity factor versus phase boundary thickness for $\tilde{j}_{0}=1$ and different values of dimensionless surface stress and interaction parameter. It is evident that, a higher value of $\tilde{\omega}$, results in a higher stress intensity factor. It is because of the fact that when $\tilde{\omega}$ is increased, the concentrations corresponding to the minimum of homogeneous free energy deviate from each other [42]. In other words, the difference between two equilibrium concentrations in each phase is increased resulting in higher tensile stresses and stress intensity factor because of the direct relationship between stress and stress intensity factor. On the other hand, as discussed in the previous section, when $\tau^{*}$ increases, the tensile stresses in the width of the particle reduce, and therefore the stress intensity factor decreases according to Eq. (36). 


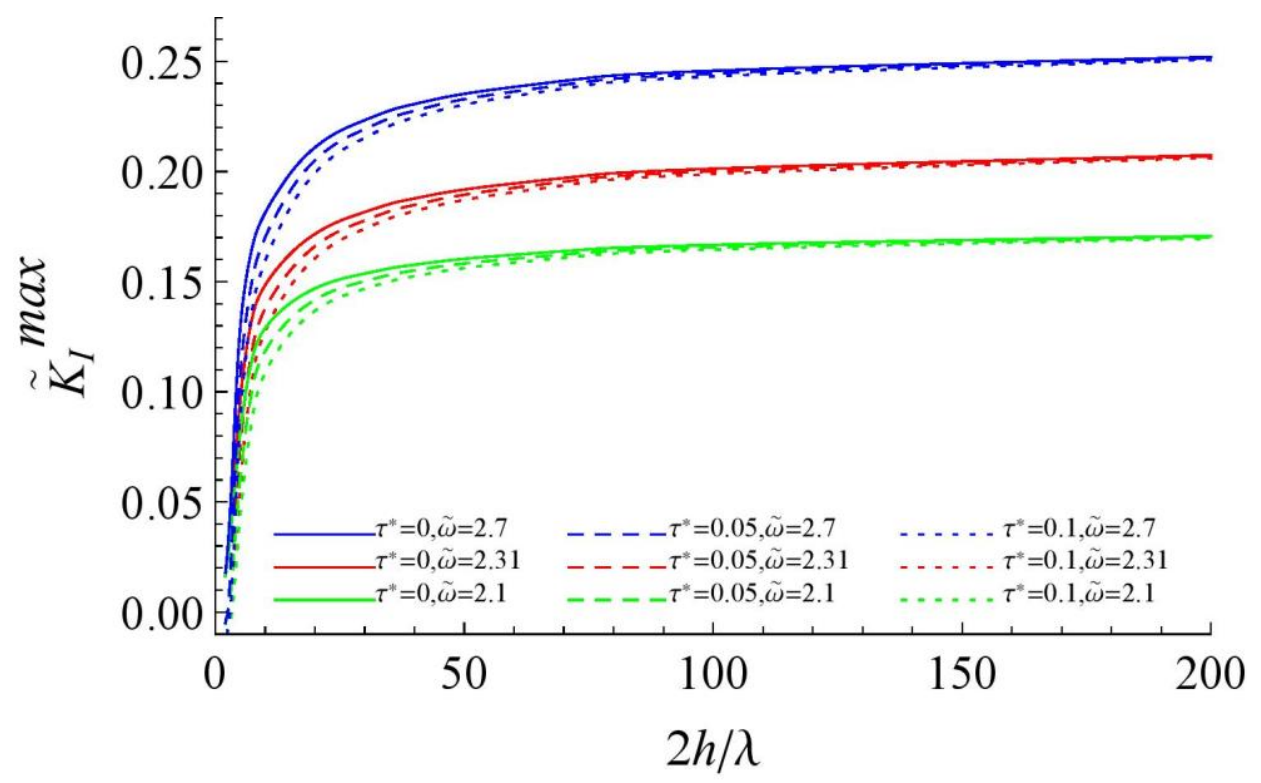

Fig. 14: Maximum stress intensity factor versus phase boundary thickness parameter for $\tilde{j}_{0}=1$, different possible values of dimensionless surface stress and interaction parameter

Figure 15 shows the stress intensity factor versus phase boundary thickness parameter for $\tilde{\omega}=2.31$ and different values of dimensionless surface stress and flux. As can be seen, once the $\tilde{j}_{0}$ value is increased, the stress intensity factor increases as well. In fact, when $\tilde{j}_{0}$ is increased, the release of concentrations from a specific surface occurs more quickly [42], which is reflected in sharper concentration and stress profiles. Subsequently, due to the direct relation between stresses and stress intensity factor (Eq. (36)), the stress intensity factor increases accordingly. On the other hand, as discussed in the previous section, higher values of $\tau^{*}$ mitigates the tensile stresses, subsequently, the stress intensity factor reduces based on Eq. (36). 


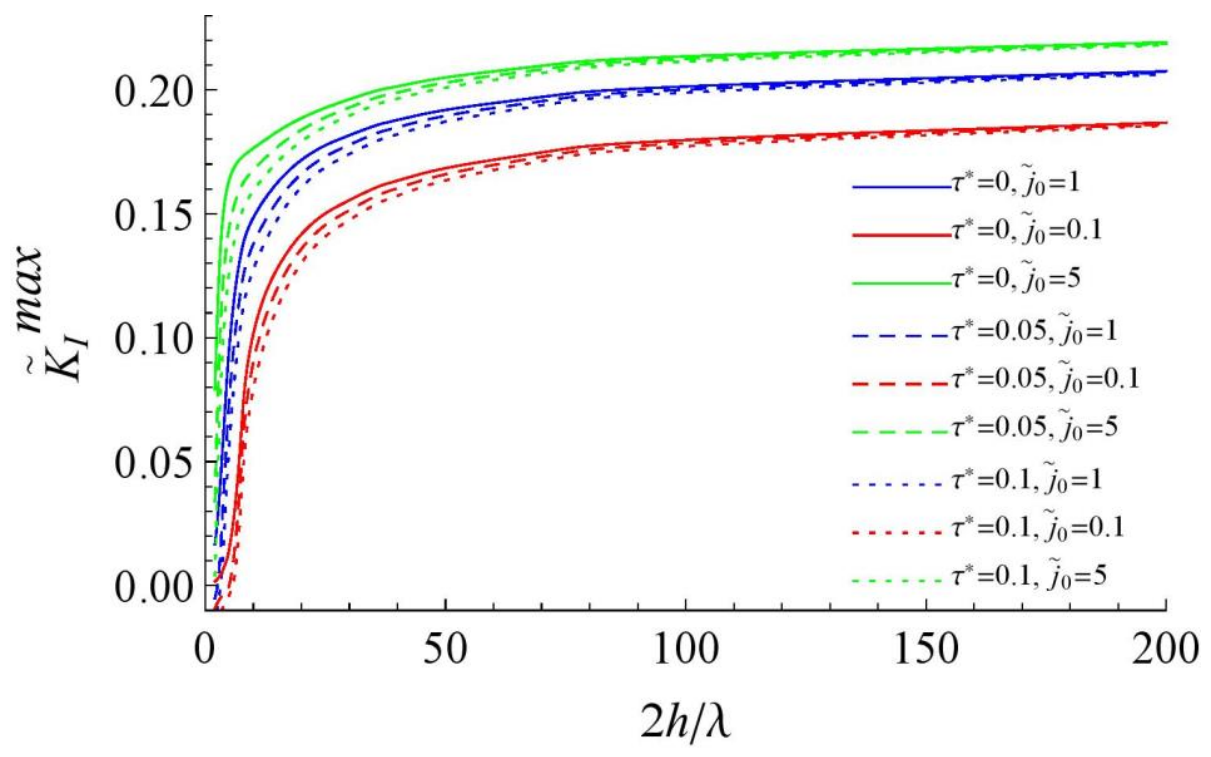

Fig. 15: Maximum stress intensity factor versus phase boundary thickness parameter for $\tilde{\omega}=2.31$ and different possible values of dimensionless surface stress and flux values

Fig 14 and 15 demonstrate that the phase-separating parameters such as the flux and interaction parameter are more influential on the stress intensity factor than surface mechanics parameters such as dimensionless surface stress and surface modulus.

\subsection{Energy function}

In this section, the Cahn-Hilliard function consisting of two terms with respect to the average concentration is plotted for different values of electrode thickness $(h)$.

As shown in Fig. 16, when the flux is equal to 0.01, the energy diagram corresponding to the lowest gradient energy term $\left(f_{g}\right)$ or the highest value for $h$ in which $h=100 \lambda$ lacks extrema and is almost linear between the two equilibrium concentration values. This suggests that, when the effect of gradient energy is negligible compared to homogeneous free energy, and the electrode thickness value, $h$, is large enough, there is no high phase-separation in the system. Therefore, there is no extrema concerning the two equilibrium concentrations. On the other hand, as $h$ 
decreases, the corresponding diagrams exhibit two minimum and one maximum between the two equilibrium concentration values. The trend concluded is similar to that reported by Burch et al. [54] and Huttin and Kamlah [73], even though the homogeneous free energy function in Ref. [76] is not symmetric; consequently, the diagram is not symmetric. In addition, as shown in Fig. 17, when the flux dimensionless value is selected as unity, the energy diagram shifts to the left for high values of $h$, whereas it tends to demonstrate a symmetric shape with two minimums and one maximum for small values of $h$. This highlights the effect of surface flux for high thickness values $h$, which causes an asymmetric diagram and forces the system to have unbalanced energies in two phases.

By comparing Fig. 16 with Fig. 17, it can be inferred that an increase in flux exacerbates the phenomenon of phase-separation and introduces unbalanced energies in the system.

As mentioned in the concentration profiles section, if the electrode thickness is small enough compared to phase boundary thickness, the phase-separation phenomenon is suppressed (nanoconfinement effect), in which the energy diagram for $\lambda=h$ without phase-separation will be the same as the homogeneous energy diagram. 


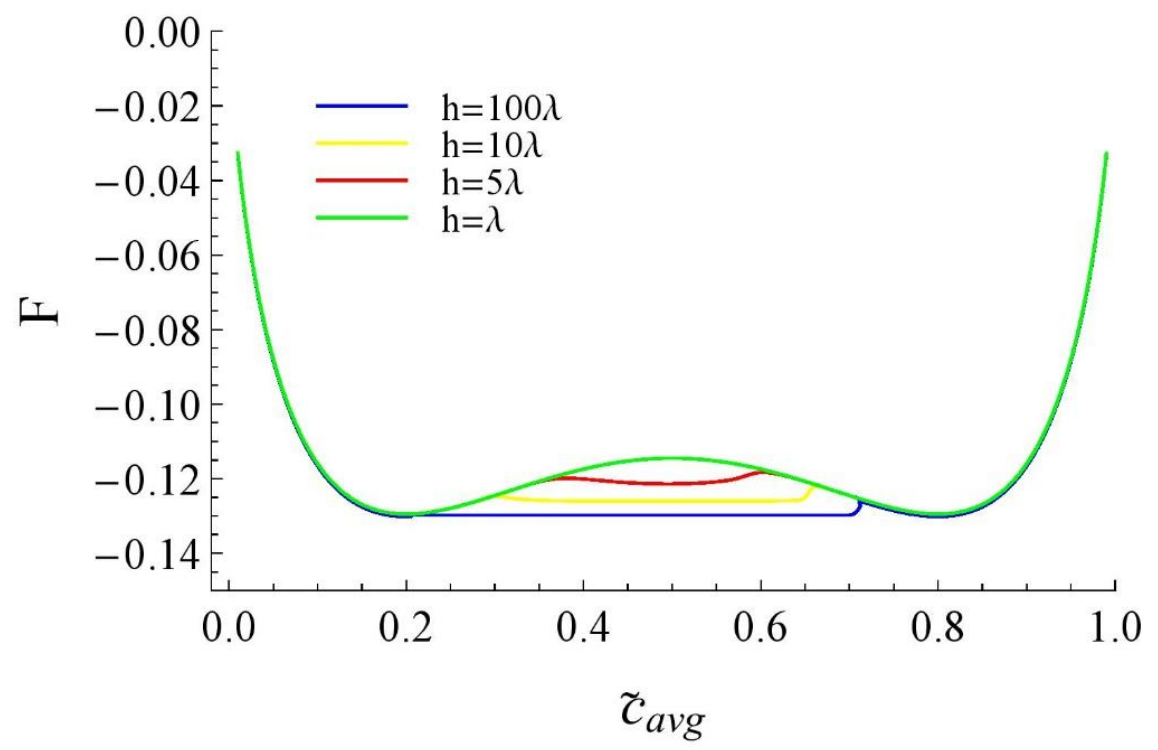

Fig. 16: Total energy $\int_{V}\left(f_{h}+f_{g}\right) d V$ versus the average lithium concentration $\tilde{c}=\int \frac{c d x}{c}$ in a planar electrode particle for different values of $h$ and $\tilde{j}_{0}=0.01$

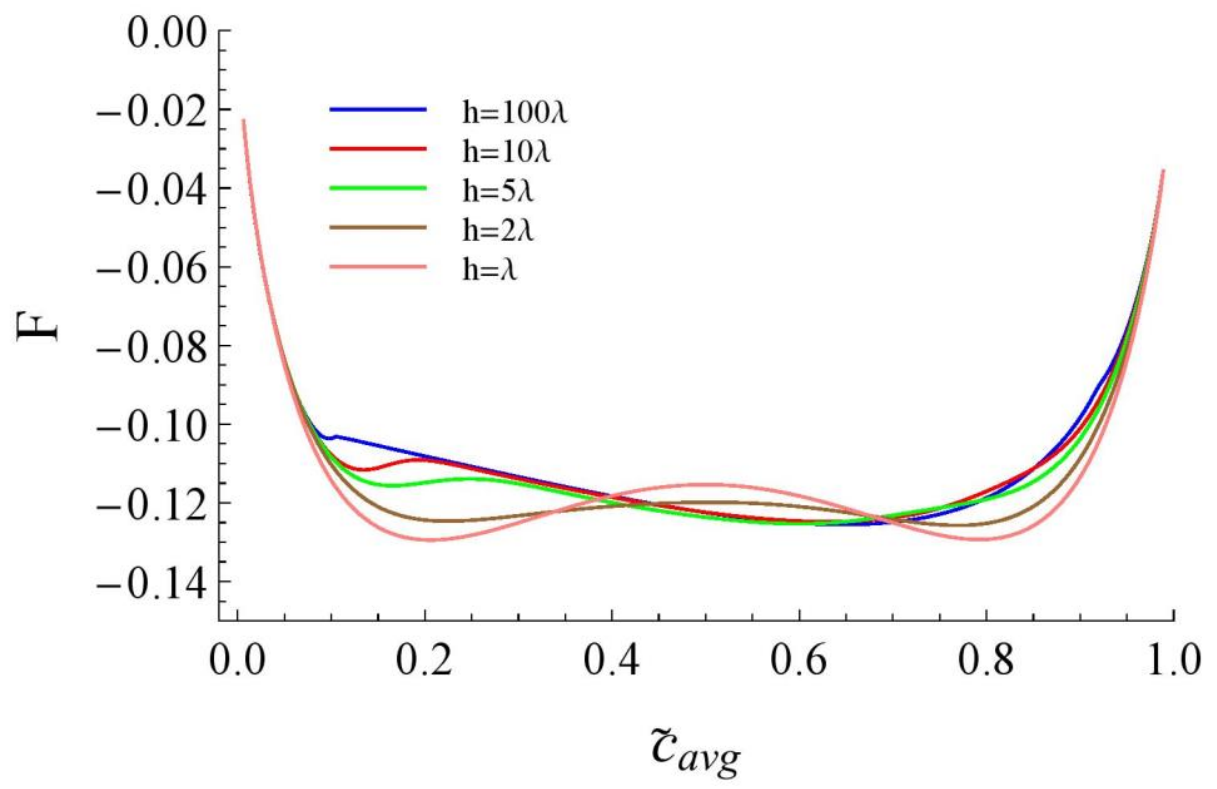

Fig. 17: Total energy $\int_{V}\left(f_{h}+f_{g}\right) d V$ versus the average lithium concentration $\tilde{c}=\int \frac{c d x}{c}$ in a planer electrode particle for different values of $h$ and $\tilde{j}_{0}=1$ 


\section{Conclusion}

Understanding the mechanical and degradation mechanisms in lithium-ion batteries has been become a big challenge since such mechanisms are in direct correlation with the lifetime of these batteries. Experiments show that mechanical behavior of nanostructures cannot appropriately be characterized by classical continuum theories. Therefore, in this paper, the surface stress effects on the fracture mechanics behavior of phase-separating planar electrodes is investigated by using the surface stress theory, a suitable method for nanostructures. The following results for both classical and phase-separating diffusion methods are obtained:

- Two surface stress parameters cannot affect the concentration profile since the coupling effect of stress and diffusion has been ignored.

- The diffusion-induced stresses and the stress intensity factor reduce when the surface stress parameters are positive.

- As the phase boundary thickness increases, i.e., the phase separation phenomenon is weakened, the effects of surface mechanics parameters become more obvious.

- The dimensionless surface parameter has a stronger effect on the diffusion-induced stresses and stress intensity factor in comparison with the surface modulus parameter.

- The phase-separating parameters such as the interaction parameter and flux are the primary decisive factors compared to surface mechanics parameters in diffusion-induced stresses and stress intensity factor.

- The comparison between two different modellings of surface constitutive equation was made based on elastic strain and total strain. It is shown that at the beginning of the process, the diffusion-induced stresses for the elastic strain based model are about $2 \%$ higher than 
the corresponding values in the total strain case, however, the differences between these two cases decreases over time.

- As the gradient energy coefficient increases, the interaction energy becomes more influential and the energy function diagram shows two minima and one maxima between two equilibrium concentrations.

- As the flux increases, the diagram shifts to the left for small values of $\tilde{\lambda}$, whereas when $\tilde{\lambda}$ equals unity the diagram becomes more symmetric and exhibits two minima and one maxima.

The presented method in the paper can be beneficial in providing a more accurate analysis of fracture mechanics of phase-separating planar electrodes in the presence of surface stress so that the parameters $\tau^{*}$ and $k^{*}$ can be predicted accurately.

For future studies, it is recommended to consider the effects of surface stress on crack faces. In addition, the effects of coating on the particle, inhomogeneity and anisotropy of the material can be considered.

\section{References}

[1] A. Mukhopadhyay and B. W. Sheldon, "Deformation and stress in electrode materials for Li-ion batteries," Prog. Mater. Sci., vol. 63, no. January, pp. 58-116, 2014.

[2] I. Ryu, J. W. Choi, Y. Cui, and W. D. Nix, "Size-dependent fracture of Si nanowire battery anodes," J. Mech. Phys. Solids, 2011.

[3] H. Haftbaradaran, X. Xiao, M. W. Verbrugge, and H. Gao, "Method to deduce the critical size for interfacial delamination of patterned electrode structures and application to lithiation of thin-film silicon islands," J. Power Sources, vol. 206, pp. 357-366, 2012.

[4] H. Haftbaradaran and H. Gao, "Ratcheting of silicon island electrodes on substrate due to 
cyclic intercalation," Appl. Phys. Lett., vol. 100, no. 12, p. 121907, Mar. 2012.

[5] M. A. Rahman, G. Song, A. I. Bhatt, Y. C. Wong, and C. Wen, "Nanostructured Silicon Anodes for High-Performance Lithium-Ion Batteries," Adv. Funct. Mater., vol. 26, no. 5, pp. 647-678, Feb. 2016.

[6] M. Feng, J. Tian, H. Xie, Y. Kang, and Z. Shan, "Nano-silicon/polyaniline composites with an enhanced reversible capacity as anode materials for lithium ion batteries," J. Solid State Electrochem., vol. 19, no. 6, pp. 1773-1782, Jun. 2015.

[7] Z.-L. Xu, X. Liu, Y. Luo, L. Zhou, and J.-K. Kim, "Nanosilicon anodes for high performance rechargeable batteries," Prog. Mater. Sci., vol. 90, pp. 1-44, Oct. 2017.

[8] A. W. McFarland and J. S. Colton, "Role of material microstructure in plate stiffness with relevance to microcantilever sensors," J. Micromechanics Microengineering, vol. 15, no. 5, pp. 1060-1067, May 2005.

[9] D. C. C. Lam, F. Yang, A. C. M. Chong, J. Wang, and P. Tong, "Experiments and theory in strain gradient elasticity," J. Mech. Phys. Solids, vol. 51, no. 8, pp. 1477-1508, Aug. 2003.

[10] Eringen, A. Cemal, and D. G. B. Edelen. "On nonlocal elasticity." International Journal of Engineering Science 10.3 (1972): 233-248

[11] R. D. Mindlin and H. F. Tiersten, "Effects of couple-stresses in linear elasticity," Arch. Ration. Mech. Anal., vol. 11, no. 1, pp. 415-448, 1962.

[12] Eringen, A. Cemal. "Linear theory of micropolar elasticity." Journal of Mathematics and Mechanics (1966): 909-923.

[13] N. A. Fleck and J. W. Hutchinson, "A phenomenological theory for strain gradient effects in plasticity," J. Mech. Phys. Solids, vol. 41, no. 12, pp. 1825-1857, Dec. 1993. 
[14] M. E. Gurtin and A. Ian Murdoch, "Surface stress in solids," Int. J. Solids Struct., vol. 14, no. 6 , pp. $431-440,1978$.

[15] M. E. Gurtin and A. Ian Murdoch, "A continuum theory of elastic material surfaces,” Arch. Ration. Mech. Anal., vol. 57, no. 4, pp. 291-323, Dec. 1975.

[16] S. Prussin, "Generation and Distribution of Dislocations by Solute Diffusion," J. Appl. Phys., vol. 32, no. 10, pp. 1876-1881, Oct. 1961.

[17] J. Christensen and J. Newman, "A Mathematical Model of Stress Generation and Fracture in Lithium Manganese Oxide,” J. Electrochem. Soc., vol. 153, no. 6, p. A1019, 2006.

[18] Y. T. Cheng and M. W. Verbrugge, "Evolution of stress within a spherical insertion electrode particle under potentiostatic and galvanostatic operation," J. Power Sources, 2009.

[19] X. Zhang, W. Shyy, and A. Marie Sastry, "Numerical Simulation of Intercalation-Induced Stress in Li-Ion Battery Electrode Particles," J. Electrochem. Soc., vol. 154, no. 12, p. S21, 2007.

[20] Y. Peng, K. Zhang, B. Zheng, and F. Yang, "Semi-analytical solution of lithiation-induced stress in a finite cylindrical electrode," J. Energy Storage, vol. 25, p. 100834, Oct. 2019.

[21] T. Ohzuku, "Electrochemistry of Manganese Dioxide in Lithium Nonaqueous Cell," J. Electrochem. Soc., vol. 137, no. 3, p. 769, 1990.

[22] W. Liu, "Mechanism of the Electrochemical Insertion of Lithium into LiMn2O4 Spinels," J. Electrochem. Soc., vol. 145, no. 2, p. 459, 1998.

[23] A. Van der Ven, "Phase transformations and volume changes in spinel LixMn2O4," Solid State Ionics, vol. 135, no. 1-4, pp. 21-32, Nov. 2000.

[24] W. H. Woodford, Y.-M. Chiang, and W. C. Carter, "Electrochemical Shock in Ion- 
Intercalation Materials with Limited Solid-Solubility," J. Electrochem. Soc., vol. 160, no. 8, pp. A1286-A1292, 2013.

[25] J. Park, W. Lu, and A. M. Sastry, "Numerical Simulation of Stress Evolution in Lithium Manganese Dioxide Particles due to Coupled Phase Transition and Intercalation," J. Electrochem. Soc., vol. 158, no. 2, p. A201, 2011.

[26] R. Deshpande, Y.-T. Cheng, M. W. Verbrugge, and A. Timmons, "Diffusion Induced Stresses and Strain Energy in a Phase-Transforming Spherical Electrode Particle," J. Electrochem. Soc., vol. 158, no. 6, p. A718, 2011.

[27] Y. C. Song, Z. Z. Li, A. K. Soh, and J. Q. Zhang, "Diffusion of lithium ions and diffusioninduced stresses in a phase separating electrode under galvanostatic and potentiostatic operations: Phase field simulations,” Mech. Mater., vol. 91, pp. 363-371, 2015.

[28] Y. Xie, M. Qiu, X. Gao, D. Guan, and C. Yuan, "Phase field modeling of silicon nanowire based lithium ion battery composite electrode," Electrochim. Acta, vol. 186, pp. 542-551, Dec. 2015.

[29] X. Gao, D. Fang, and J. Qu, “A chemo-mechanics framework for elastic solids with surface stress," Proc. R. Soc. A Math. Phys. Eng. Sci., vol. 471, no. 2182, p. 20150366, Oct. 2015.

[30] Y.-T. Cheng and M. W. Verbrugge, "The influence of surface mechanics on diffusion induced stresses within spherical nanoparticles," J. Appl. Phys., vol. 104, no. 8, p. 083521, Oct. 2008.

[31] R. Deshpande, Y.-T. Cheng, and M. W. Verbrugge, "Modeling diffusion-induced stress in nanowire electrode structures," J. Power Sources, vol. 195, no. 15, pp. 5081-5088, Aug. 2010.

[32] F. Hao, X. Gao, and D. Fang, "Diffusion-induced stresses of electrode nanomaterials in 
lithium-ion battery: The effects of surface stress," J. Appl. Phys., vol. 112, no. 10, p. 103507, Nov. 2012.

[33] F. Hao and D. Fang, "Diffusion-Induced Stresses of Spherical Core-Shell Electrodes in Lithium-Ion Batteries: The Effects of the Shell and Surface/Interface Stress," J. Electrochem. Soc., vol. 160, no. 4, pp. A595-A600, Feb. 2013.

[34] Y. Liu, P. Lv, J. Ma, R. Bai, and H. L. Duan, "Stress fields in hollow core-shell spherical electrodes of lithium ion batteries," Proc. R. Soc. A Math. Phys. Eng. Sci., vol. 470, no. 2172, pp. 20140299-20140299, Oct. 2014.

[35] X. Zhang, H.-S. Chen, and D. Fang, "Effects of surface stress on lithium-ion diffusion kinetics in nanosphere electrodes of lithium-ion batteries," Int. J. Mech. Sci., vol. 169, p. 105323, Mar. 2020.

[36] H. M. Shodja, B. Shahryari, P. Azizi, and F. Roumi, "Exact Diffusion-Induced Elastic Fields of a Spherical Core-Shell Nano-Electrode Li-Ion Battery via Spectral Theory,” J. Electrochem. Soc., vol. 167, no. 13, p. 130540, Oct. 2020.

[37] Y. Hu, X. Zhao, and Z. Suo, “Averting cracks caused by insertion reaction in lithium-ion batteries," J. Mater. Res., vol. 25, no. 06, pp. 1007-1010, 2010.

[38] K. Zhao, M. Pharr, J. J. Vlassak, and Z. Suo, "Fracture of electrodes in lithium-ion batteries caused by fast charging," J. Appl. Phys., vol. 108, no. 7, p. 073517, Oct. 2010.

[39] W. H. Woodford, W. C. Carter, and Y.-M. Chiang, "Design criteria for electrochemical shock resistant battery electrodes," Energy Environ. Sci., vol. 5, no. 7, p. 8014, 2012.

[40] W. H. Woodford, Y.-M. Chiang, and W. C. Carter, “'Electrochemical Shock' of Intercalation Electrodes: A Fracture Mechanics Analysis,” J. Electrochem. Soc., vol. 157, no. 10, pp. A1052-A1059, 2010. 
[41] K. Zhao, M. Pharr, L. Hartle, J. J. Vlassak, and Z. Suo, "Fracture and debonding in lithiumion batteries with electrodes of hollow core-shell nanostructures," J. Power Sources, vol. 218, pp. 6-14, Nov. 2012.

[42] B. Chen, J. Zhou, and R. Cai, "Analytical model for crack propagation in spherical nano electrodes of lithium-ion batteries," Electrochim. Acta, vol. 210, pp. 7-14, Aug. 2016.

[43] H. Haftbaradaran, A. Maddahian, and F. Mossaiby, "A fracture mechanics study of the phase separating planar electrodes: Phase field modeling and analytical results," J. Power Sources, 2017.

[44] S. Esmizadeh, H. Haftbaradaran, and F. Mossaiby, "An investigation of the critical conditions leading to deintercalation induced fracture in two-phase elastic electrode particles using a moving interphase core-shell model," Eur. J. Mech. - A/Solids, vol. 74, pp. 96-111, Mar. 2019.

[45] N. Movahedi, A. Geravand, and H. Haftbaradaran, "Investigating inhomogeneity effects on the crack driving force in two-phase electrode particles using a planar composite core-shell model," Mech. Mater., vol. 152, p. 103652, Jan. 2021.

[46] X. Hu, Y. Zhao, R. Cai, and J. Zhou, "Surface effected fracture behavior of nano-spherical electrodes during lithiation reaction," Mater. Sci. Eng. A, vol. 707, pp. 92-100, Nov. 2017.

[47] C. Xu, L. Weng, B. Chen, J. Zhou, and R. Cai, "An analytical model for the fracture behavior in hollow cylindrical anodes," Int. J. Mech. Sci., vol. 157-158, pp. 87-97, Jul. 2019.

[48] Crank, John. The mathematics of diffusion. Oxford university press, 1979.

[49] B. C. Han, A. Van der Ven, D. Morgan, and G. Ceder, "Electrochemical modeling of intercalation processes with phase field models," Electrochim. Acta, vol. 49, no. 26, pp. 
4691-4699, Oct. 2004.

[50] M. . Levi and D. Aurbach, "Frumkin intercalation isotherm - a tool for the description of lithium insertion into host materials: a review," Electrochim. Acta, vol. 45, no. 1-2, pp. 167-185, Sep. 1999.

[51] J. W. Cahn and J. E. Hilliard, "Free Energy of a Nonuniform System. I. Interfacial Free Energy,” J. Chem. Phys., vol. 28, no. 2, pp. 258-267, Feb. 1958.

[52] G. K. Singh, G. Ceder, and M. Z. Bazant, "Intercalation dynamics in rechargeable battery materials: General theory and phase-transformation waves in LiFePO4," Electrochim. Acta, vol. 53, no. 26, pp. 7599-7613, Nov. 2008.

[53] M. Tang, H.-Y. Huang, N. Meethong, Y.-H. Kao, W. C. Carter, and Y.-M. Chiang, "Model for the Particle Size, Overpotential, and Strain Dependence of Phase Transition Pathways in Storage Electrodes: Application to Nanoscale Olivines," Chem. Mater., vol. 21, no. 8, pp. 1557-1571, Apr. 2009.

[54] P. Bai, D. A. Cogswell, and M. Z. Bazant, "Suppression of Phase Separation in LiFePO4 Nanoparticles During Battery Discharge," Nano Lett., vol. 11, no. 11, pp. 4890-4896, Nov. 2011.

[55] M. Z. Bazant, "Theory of Chemical Kinetics and Charge Transfer based on Nonequilibrium Thermodynamics," Acc. Chem. Res., vol. 46, no. 5, pp. 1144-1160, May 2013.

[56] D. Burch and M. Z. Bazant, "Size-Dependent Spinodal and Miscibility Gaps for Intercalation in Nanoparticles," Nano Lett., vol. 9, no. 11, pp. 3795-3800, Nov. 2009.

[57] D. A. Cogswell and M. Z. Bazant, "Coherency Strain and the Kinetics of Phase Separation in LiFePO 4 Nanoparticles," ACS Nano, vol. 6, no. 3, pp. 2215-2225, Mar. 2012.

[58] J. W. Gibbs, The Scientific Papers of J. Willard Gibbs_Longnans-Green, London, 1906_, 
Vol. 1, p. 55.

[59] R. C. Cammarata, "Surface and interface stress effects in thin films," Prog. Surf. Sci., vol. 46, no. 1, pp. 1-38, May 1994.

[60] P. MULLER, "Elastic effects on surface physics," Surf. Sci. Rep., vol. 54, no. 5-8, pp. 157258, Aug. 2004.

[61] A. RUSANOV, "Surface thermodynamics revisited," Surf. Sci. Rep., vol. 58, no. 5-8, pp. 111-239, Sep. 2005.

[62] F. D. Fischer, T. Waitz, D. Vollath, and N. K. Simha, "On the role of surface energy and surface stress in phase-transforming nanoparticles," Prog. Mater. Sci., vol. 53, no. 3, pp. 481-527, Mar. 2008.

[63] R. Shuttleworth, “The Surface Tension of Solids," Proc. Phys. Soc. Sect. A, vol. 63, no. 5, pp. 444-457, May 1950.

[64] R. E. Miller and V. B. Shenoy, "Size-dependent elastic properties of nanosized structural elements," Nanotechnology, vol. 11, no. 3, pp. 139-147, Sep. 2000.

[65] P. Sharma, S. Ganti, and N. Bhate, "Effect of surfaces on the size-dependent elastic state of nano-inhomogeneities," Appl. Phys. Lett., vol. 82, no. 4, pp. 535-537, Jan. 2003.

[66] R. C. Cammarata, K. Sieradzki, and F. Spaepen, "Simple model for interface stresses with application to misfit dislocation generation in epitaxial thin films," J. Appl. Phys., vol. 87, no. 3, pp. 1227-1234, Feb. 2000.

[67] M. Z. Mughal, R. Moscatelli, H.-Y. Amanieu, and M. Sebastiani, "Effect of lithiation on micro-scale fracture toughness of LixMn2O4 cathode," Scr. Mater., vol. 116, pp. 62-66, Apr. 2016.

[68] Y. Qi, L. G. Hector, C. James, and K. J. Kim, "Lithium Concentration Dependent Elastic 
Properties of Battery Electrode Materials from First Principles Calculations," J. Electrochem. Soc., vol. 161, no. 11, pp. F3010-F3018, Aug. 2014.

[69] J. C. Hunter, "Preparation of a new crystal form of manganese dioxide: $\lambda$-MnO2," J. Solid State Chem., vol. 39, no. 2, pp. 142-147, Sep. 1981.

[70] M. M. Thackeray, P. J. Johnson, L. A. de Picciotto, P. G. Bruce, and J. B. Goodenough, "Electrochemical extraction of lithium from LiMn2O4," Mater. Res. Bull., vol. 19, no. 2, pp. 179-187, Feb. 1984.

[71] T. L. Anderson, Fracture Mechanics: Fundamentals and Applications, 3rd ed. Taylor \& Francis, Boca Rotan, Florida. 2005.

[72] Bueckner, H. F. "Novel principle for the computation of stress intensity factors."Zeitschriftfuer Angewandte Mathematik \& Mechanik 50.9 (1970).

[73] J. R. Rice, "Some remarks on elastic crack-tip stress fields," Int. J. Solids Struct., vol. 8, no. 6, pp. 751-758, Jun. 1972.

[74] H. Tada, P.C. Paris, G.R. Irwin, The Stress Analysis of Cracks Handbook, third ed., ASME Press, New York, 2000.

[75] T. Fett, Stress Intensity Factors, T-stresses, Weight Functions: Supplement Volume, Universit€atsverlag Karlsruhe, Karlsruhe, 2009.

[76] M. Huttin and M. Kamlah, "Phase-field modeling of stress generation in electrode particles of lithium ion batteries," Appl. Phys. Lett., vol. 101, no. 13, p. 133902, Sep. 2012. 